\title{
The Craik-O'Brien-Cornsweet effect: New varieties and their theoretical implications
}

\author{
DEJAN TODOROVIĆ \\ Boston University, Boston, Massachusetts, \\ and Univerzitet u Beogradu, Belgrade, Yugoslavia
}

\begin{abstract}
New visual displays related to the Craik-O'Brien-Cornsweet effect are presented and their relevance for theories of the effect is assessed. The displays include a luminance cusp that does not induce the standard effect, an illusory chessboard, a brightness variant of the impossible staircase, and a display in which brightness varies with stimulus motion. Theories of the effect are grouped within the nonisomorphistic, the cognitive, and the mechanistic approaches. The nonisomorphistic approach is criticized as being seriously incomplete. Existing cognitively oriented proposals are criticized as inadequate. Mechanistic theories are divided into integration theories, which model the visual processing stages with standard mathematical operations such as differentiation and integration, and filling-in theories, which stress the processing dynamics of interconnected neural networks. The reported variations of the Craik-O'Brien-Cornsweet effect support the latter class of theories.
\end{abstract}

This paper presents a critical review of the main theoretical approaches to the Craik-O'Brien-Cornsweet effect (COCE), and enlarges the data base of this phenomenon by introducing several visual displays in which the luminance distributions characteristic of the COCE are used as components of more complex visual situations. These new varieties of the COCE demonstrate its dependence on the visual context, and thus provide constraints on the theories of the effect. The presentation and discussion of the displays is interleaved with the review, in order to point out more clearly their relevance to various theories.

The paper is divided into four parts. Part 1 presents the basic form of the COCE and then a standard analysis of the effect. This analysis is criticized as being unable to provide a satisfactory account of the appearance of the phenomenon. The following three parts of the paper discuss different theoretical attempts to explain the structure of the brightness percept in the COCE. The explanations are grouped under the headings of the nonisomorphistic, the cognitive, and the mechanistic approaches. The theories within these approaches vary widely in content, generality, and depth of analysis. Some refer only to the COCE; others are more general and invoke this effect as an illustration of mechanisms proposed to explain a broader class of visual phenomena. In addition, some proposals are full-fledged mathematically specified theories, whereas others give only directions in which the explanation should be sought. The variety of the approaches reflects the interest in and the importance of the phenomenon.

This work was supported by the U.S. Army Research Office (ARO DAAG-29-85-K-0095). Special thanks are extended to Ennio Mingolla for his generous help, advice, and criticism through all stages of this work. The author's mailing address is Filozofski Fakultet, Cika Ljubina 18-20, 11000 Belgrade, Yugoslavia.
The displays presented in this paper were generated on a computer-controlled graphics TV monitor. This is a much more flexible technique for the creation of variations of the COCE than the usual one involving rotating painted disks (Cornsweet, 1970). Because of the possible distortions introduced by the photographic process, the descriptions of the luminance distributions apply, strictly speaking, only to the monitor, so that the photographs in this paper serve only as illustrations.

\section{PART 1 \\ THE PHENOMENON}

\section{Two Types of Luminance-Brightness Mismatch}

A task for theories of achromatic brightness perception is to predict and explain the perceived brightness level of portions of the visual field. A basic datum for such theories is the relation of luminance, that is, the amount of visually effective light arriving at a retinal area, and brightness or lightness, which is a perceptual attribute varying from dark to bright, or from black to white. The distinction between concepts of brightness and lightness is not relevant for the present discussion because display conditions do not call for assessment of surface reflectance or illumination variables.

Experiments involving a single homogeneous patch of light on a dark surround indicate that the relationship between luminance and brightness may be modeled by a power function (Stevens, 1957). An extrapolation of this result to more complex visual situations predicts that the relative brightness levels of different regions should be proportional to power functions of their luminances. Other things being equal, each luminance level should correspond to one and only one brightness level. This prediction fails in at least two types of visual situations, cor- 
responding to the two ways in which a one-to-one relationship, such as the power function, can be violated.

The first, many-to-one type of luminance-brightness relationship is exemplified in the phenomenon of brightness constancy. It involves surfaces of equal reflectance appearing similarly bright, despite being differentially illuminated (Katz, 1935). It is usually pointed out that, in contrast to this "unorderly" many-to-one luminance-tobrightness relationship, there is generally a very orderly one-to-one reflectance-to-brightness relationship in these cases.

However, order is not so easily restored in the second, one-to-many case of luminance-brightness mismatch. In such visual situations, portions of surfaces having identical luminances (and identical reflectances) are perceived to be differentially bright. Examples include the effects of simultaneous brightness contrast (Heinemann, 1972), brightness assimilation (Helson, 1963), the Mach bands (Ratliff, 1965), the Hermann grid (Spillmann \& Levine, 1971), the Wertheimer-Benary figure (Benary, 1924/1938), the Koffka-Benussi ring (Koffka, 1935), the Ehrenstein illusion (Ehrenstein, 1941), the subjective contour figures (Kanizsa, 1979), the grating brightness effects (Quinn, 1985), the orientation-sensitive brightness effects (McCourt, 1982; White, 1979), and the brightness effects related to perceived depth (Gilchrist, 1977).

\section{The Standard Form of the Phenomenon}

The COCE also belongs to this second group of phenomena (Cornsweet, 1970; Craik, 1940; O'Brien, 1958). One variation of this phenomenon is shown in Plate 1. It consists of two vertically oriented rectangular regions on a dark background. There is a relatively small but clearly perceivable brightness difference between the two rectangles. The two regions have fairly homogeneous brightness levels, except perhaps for very narrow stripes, reminiscent of Mach bands, located at their shared border. Such a percept might be caused by a luminance step, that is, two adjoining surfaces having different homogeneous luminance levels. The profile of a luminance step is shown in Figure 1a. However, the horizontal cross-section of the luminance profile of the two rectangles in Plate 1 is not the step, but the cusp-shaped distribution in Figure $1 b$. The luminance over the two rectangles is identical, except for two relatively narrow regions containing luminance gradients flanking the common border of the rectangles. The left gradient is positive, that is, its luminance increases toward the center, and the right one is negative. The horizontal spatial extent of either gradient in Plate 1 is approximately one fifth of the horizontal side of either rectangle. Unlike the luminance gradients, the difference in brightness is not confined to the border region, but extends over the entire area of the rectangles.

The display looks most like a real step if the viewing distance is small, say $10 \mathrm{~cm}$. Larger viewing distances induce smaller retinal extents and larger slopes of the luminance gradients. The perceptual consequence is that the brightness difference between the two rectangles de-

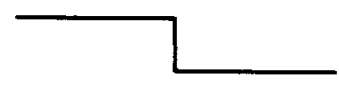

(a)

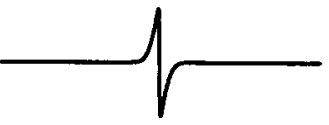

(c)

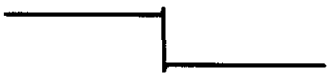

(e)

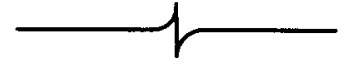

(b)

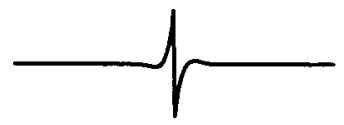

(d)

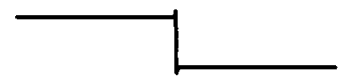

(f)
Figure 1. Six distributions involved in the analysis of the COCE. The first row $(a, b)$ contains two luminance distributions, the second row (c, $d$ ) contains the two corresponding distributions of neural activity, and the third row (e, f) contains the two induced brightness distributions. The left column refers to the luminance step distribution (a), and its physiological (c) and perceptual (e) counterpart, respectively. The right column presents the luminance cusp distribution (b), and its physiological (d) and perceptual (f) counterpart. The physiological distributions refer to the patterns of activity induced by the luminance distribution in a layer of on-center/offsurround cells.

creases, and the brightness gradients flanking the step become increasingly apparent. Prolonged fixation tends to enhance the COCE. The brightness difference between the two rectangles can be shown to depend on the luminance conditions at the border region by simply occluding the border, for example with a pencil or even a piece of thin wire: the two rectangles will then be perceived as equally bright, in accordance with their equal luminance levels. Removing the occlusion reinstantiates the brightness difference, after a period of short but noticeable delay. It should be noted that although this demonstration of the illusory nature of the COCE is perceptually compelling, the brightness percept of the occluded display may not have a simple explanation. The reason is that brightness equality is induced by border occlusion in two different visual situations. It is induced in cases of luminance cusps, such as Plate 1 , but it is also induced in cases of ordinary luminance steps, provided that the luminance difference between two sides of the step is small (Tolhurst, 1972). Thus border occlusion transforms an illusory percept into a veridical one, in the case of luminance cusps, but has the opposite effect in the case of small luminance steps.

Parametric studies of the COCE have investigated its dependence on several factors, including viewing distance and total stimulus extent (Békésy, 1972), average luminance level (Heggelund \& Krekling, 1976), luminance 
contrast and extent of the flanking gradients (Dooley \& Greenfield, 1977; Growney \& Neri, 1986; Isono, 1979a), and gradient polarity (Hamada, 1982, 1985). A related phenomenon involving square-wave gratings without the fundamental component is the "missing fundamental illusion"' (Campbell, Howell, \& Johnstone, 1978; Isono, 1979b; Maffei, Morrone, Pirchio, \& Sandini, 1979; Sakata, 1981; Sullivan \& Georgeson, 1977). Chromatic phenomena analogous to the COCE were studied by Arend (1973), S. S. Stevens and E. Land (see Land, 1974), van den Brink and Keemink (1976), and Ware and Cowan (1983).

\section{An Explanation and a Problem}

Why does local brightness not correspond to local luminance in Plate 1? Why do two different luminance distributions (Figures $1 \mathrm{a}$ and $1 \mathrm{~b}$ ) induce two very similar brightness distributions (Figures le and 1f)? Profiles 1a and $\mathbf{l b}$ are similar in that they both contain an abrupt luminance step, but are different in the gradual manner of change of luminance in the areas flanking the step. In terms of Fourier analysis, the two distributions have similar high-frequency content but different low-frequency components. However, the visual system is relatively insensitive to low-spatial-frequency stimulation (Campbell \& Robson, 1968). According to Cornsweet (1970), Campbell et al. (1978), Ratliff (1978), and Ratliff and Sirovich (1978), these facts amount to an explanation of the COCE. The cusp-shaped and the step-shaped distributions look similar because their effects are similar: the visual system suppresses the aspects of these stimuli that differ (shallow spatial variation of luminance), and transmits more faithfully the attribute they have in common (abrupt change). This is an elegant reduction of a surprising phenomenon to well-known facts.

However, it can be argued that this explanation is incomplete, since it does not seem to account for the structure of the appearance of the stimulus. The problem is that there is a mismatch between the shape of the brightness profile of the percept and its presumed neural counterpart (see Arend, 1973; Cohen \& Grossberg, 1984; Cornsweet, 1970; Davidson \& Whiteside, 1971). The luminance cusp distribution (Figure $1 \mathrm{~b}$ ) gives rise to a percept that has the shape of a step (Figure 1f). However, the presumed physiological foundation of the percept, according to the preceding analysis, has a quite different profile (Figure 1d), one that is more similar to the cuspshaped profile of the underlying luminance distribution.

This cusp-shaped neural distribution arises as the result of the transformation of the luminance cusp distribution through a bandpass linear filter. A very similar cuspshaped distribution (Figure 1c) results when the stepshaped luminance distribution (Figure 1a) is transformed through the same filter (Cornsweet, 1970; Ratliff \& Sirovich, 1978). The weighting function of the filter has the well-known sombrero shape. The particular weighting function that was convolved with the luminance distributions in Figure 1 is a simple difference of Gaussians
(Rodieck, 1965), but other formalizations, such as shunting equations (Grossberg, 1983) or the Laplacian of Gaussian (Marr \& Hildreth, 1980) would lead to similar results. These functions model the properties of neurons with concentric antagonistic on-center/off-surround receptive fields.

Retinal cells of this type have nonnegligible spontaneous firing levels and are relatively insensitive to homogeneous luminance distributions covering the whole receptive field (Enroth-Cugell \& Robson, 1984). These cells are maximally excited by a bright spot confined to the central position of the receptive field, and maximally inhibited by a bright annulus. However, stimulus distributions containing an abrupt luminance step, such as Figures $1 \mathrm{a}$ and $1 \mathrm{~b}$, would also cause substantial changes in the firing rate of cells whose receptive fields were in the vicinity of the step. The amount of firing-rate change would depend on the exact position of the edge with respect to the excitatory and inhibitory portions of the receptive field. These properties explain the shapes of Figures 1c and 1d. In the case of the step stimulus, direct empirical corroboration of these simulations was provided by Enroth-Cugell and Robson $(1966,1984)$. In these studies, instead of simultaneously recording the response of a large number of neurons to a static step, an experimentally much more feasible approach was used, in which the response from only one on-cell at a time was recorded but the position of the stimulus was varied. The record of the retinal response to the luminance step agrees with the shape of Figure 1c.

It is thus fairly clear how two different luminance distributions (Figures 1a and $1 \mathrm{~b}$ ) can give rise to two similar neural distributions (Figures $1 \mathrm{c}$ and 1d). However, there remains the problem of how these cusp-shaped neural distributions could provide the appropriate physiological foundation for the step-shaped perceptual brightness distributions (Figures le and 1f). How do portions of these neural distributions, which have equal activity levels, such as those located at some distance to the left and to the right from the position of the step, come to correspond to different brightness levels in the percept? A complete account of the COCE should explain how the luminance cusp distribution, Figure $1 \mathrm{~b}$, is transformed into the brightness step distribution, Figure 1f. However, what is actually offered in the preceding analysis is an account of the transformation of the luminance cusp into the neural cusp, Figure 1d; this does not seem to bring us closer to understanding the step shape of the brightness distribution. The low-frequency Fourier components may be absent from the distribution of neural activity at a visual processing level involving neurons with concentric antagonistic receptive fields; however, they are clearly present in the brightness distribution. In sum, this theory explains why luminance steps and cusps look alike, but not what they look like.

There are at least three ways of dealing with this problem, besides ignoring it. The first possibility is to assume that activity at an early level of visual processing may not 
account for all aspects of suprathreshold brightness perception. However, the early level is followed by some additional levels, so that at some later stage a neural distribution may arise that corresponds more closely to the percept. Several theories of the COCE, discussed in Part 4 of this paper, are based on this idea, which will be termed the "mechanistic" approach. The second approach is to agree that additional processing is needed, but to assume that it consists in interpretative, cognitive-like activities. Some proposals of this type, termed the "cognitive approach," will be discussed in Part 3. Finally, the "nonisomorphistic" approach, described in Part 2, denies the necessity of any additional processing stage.

\section{PART 2 \\ THE NONISOMORPHISTIC APPROACH}

\section{Formulation and Implications of the Nonisomorphistic Approach}

The guiding idea of the nonisomorphistic approach is that the similarity of profiles of neural activities induced by steps and cusps is sufficient to explain the similarity of percepts; it is not necessary to require isomorphism, that is, the identity of shapes of spatial distributions of percepts and the underlying neural activities. To postulate a cusp distribution of neural activity as the ultimate neural counterpart of a brightness step distribution is, it is claimed, conceptually parsimonious, involves no logical contradiction, and is consistent with empirical data. Ratliff and Sirovich (1978) put it this way:

The neural activity which underlies appearance must reach a final stage eventually. It may well be that marked neural activity adjacent to the edges (as is postulated in this model and is commonly observed in neurophysiological experiments) is, at some level of the visual system, that final stage and is itself the sought-for end process. Logically, nothing more is required. Nevertheless, we cannot by any reasoning eliminate a priori some higher-order stage or filling in process. ... But parsimony demands that any such additional stage or process be considered only if neurophysiological evidence for it should appear.

Similar doubts regarding the necessity of additional processing machinery for the explanation of the COCE were also expressed by Ratliff (1978), Bridgeman (1983), Foster (1983), and Laming (1983). This approach certainly appears to be more parsimonious than any alternative explanation that calls for further transformation of activity patterns. In addition, neurophysiological data on filling-in processes do not seem to be available. However, presently this is only an interesting suggestion for the explanation of the COCE, and not yet a full-fledged theory. In the following, some of its implications will be discussed. To anticipate, I will claim that a more precise and complete formulation of such an approach is not straightforward and that its parsimony is spurious.

The issues raised by the nonisomorphistic explanation of the COCE have roots in the philosophical mind-body problem and a long history in psychology. They concern the nature of the relationship of neural activity and percepts (Brindley, 1970; Köhler, 1929; Mach, 1865; Müller, 1896; Teller, 1984). This relationship is crucial in physiological explanations of perceptual phenomena, but is seldom itself the focus of attention. It is sometimes expressed more formally in so-called "psychophysical axioms" (Müller, 1896) or "linking propositions" (Teller, 1984). These propositions link statements about perceptual states with statements about neural states. A number of issues concerning such propositions have been discussed by Teller (1984). An important concept is the "psychophysical level"' (Müller, 1896), or the "bridge locus" (Teller \& Pugh, 1983), which consists of a hypothetical set of neurons whose activity is postulated to be the ultimate physiological counterpart of a percept. This concept is probably equivalent to Ratliff and Sirovich's (1978) "final stage" and "the sought-for end process."

A linking proposition relevant to the present discussion is the statement that two structurally similar neural distributions (such as Figures 1c and 1d) are likely to induce two structurally similar perceptual distributions. This proposition explains why two neural cusps, whatever their causal histories, should look alike, but it does not imply any claim concerning the particular spatial form of neural or perceptual distributions.

Another type of linking proposition, related to the "analogy family" of Teller (1984), concerns the nature of the relationship of the structure of the neural distribution and the structure of the perceptual distribution. A number of neurophysiologically oriented theories of suprathreshold brightness phenomena assume explicitly, or more often implicitly, that this relationship is an. isomorphism. In contrast, the nonisomorphistic approach, exemplified in the quote from Ratliff and Sirovich (1978), explicitly denies the need for such an assumption.

The underlying idea of the isomorphistic approach to brightness perception is that spatial variations of brightness in the visual field should be accounted for by analogous variations of neural activity. In other words, the neural distribution should "look like" the percept. However, there is no consensus among the theories as to which neural level is responsible for the brightness percept. One candidate set consists of cells with circular concentric antagonistic receptive fields. For example, the local increments and decrements in the spatial distribution of activity in such cells are assumed to have perceptual counterparts in bright and dark Mach bands (see Cornsweet, 1970; Ratliff, 1965; Teller, 1980), in spots located at intersections of lines in the Hermann grid (Baumgartner, 1960, Spillmann \& Levine, 1971), and in the "glowing diagonals" found in some paintings by Vasarely (Hurvich, 1981). Another candidate set for neurons directly underlying the brightness percept consists of units with elongated receptive fields, such as cortical hypercomplex cells. Their activity level is assumed to correspond to the brightness intensity of percepts in the phenomena of grating induction (Foley \& McCourt, 1985), neon flanks (Redies, Spillmann, \& Kunz, 1984), and the Ehrenstein illusion (Fuld 
\& O'Donnell, 1984). Finally, in some theories, the bridge locus is a hypothetical neural level consisting of units with strong lateral facilitatory interconnections. Such units support a filling-in process, which may be modulated by stimulus-induced boundaries. Processes of this type are assumed in the explanations of brightness percepts in stabilized images and scotoma (Gerrits \& Vendrick, 1970), the simultaneous brightness contrast (Frisby, 1979), and in some theories of the COCE described in Part 4. These theories all assume that the initial cusp-shaped neural distribution is subsequently transformed into a step-shaped neural distribution, but they propose different mechanisms to implement this transformation. The step shape of the final neural disribution is assumed to explain the step shape of the brightness distribution.

A logical consequence of the isomorphistic approach is that a neural activity distribution not isomorphic with the percept cannot be its ultimate neural foundation. To illustrate this, note that it is likely that at some levels of the visual system layers of cells exist for which the response to a ramp-shaped stimulus, known to induce perceptual Mach bands, definitely does not involve neural overshoots or undershoots. For example, the shape of neural activity at the receptor level might relatively faithfully follow the ramp shape of the luminance distribution. As another example, the shape of the neurai distribution in a layer of cells with large receptive fields without inhibitory surrounds would be a strongly blurred version of the luminance distribution. The isomorphistically constrained conclusion would be that the neural distributions at those levels could not provide an explanation for Mach bands because they could not account for the perceptual overshoots and undershoots (see Teller, 1980). However, if the isomorphistic constraint is renounced, the way is open for a different conclusion: one could argue that no further processing level is logically required, that the structure of the percept could actually be explained at any of those levels, and that no special concern should be given to the mismatch of shapes of neural and brightness distributions: a neural sharp or blurred ramp-shaped distribution, or indeed a distribution of any other shape, might just happen to be the ultimate code, the final neural stage corresponding to a peceptual distribution involving Mach bands.

It is clear from this example that the negative claim ("there is no need for isomorphism") must be complemented by more positive statements concerning the specification of the proposed final neural-perceptual map, and the nature of its implementation. This map is currently specified only in that it transforms neural cusps into perceptual steps. But how should this example be extended to predict the shape of percepts corresponding to neural distributions other than the cusp? For example, given that the neural-perceptual map is nonisomorphic, would a neural distribution involving increments and decrements be predicted to induce the Mach band percept? Clearly, a general transformational principle is needed to provide a unified set of appearance predictions for a variety of neural distributions. Only within such a framework could one begin to compare the nonisomorphistic approach with the isomorphistically oriented explanations. Note that in the isomorphistic approach the corresponding problem of the specification of the final neural-perceptual map has a trivial solution: this map is well specified for any distribution, since it is always the identity function.

In addition to the specification of the form of the neuralperceptual map, the nonisomorphistic approach should also be more specific about the mechanism behind this map. As an example, consider two small portions of Plate 1, one located at the center of the left rectangle, and the other at the center of the right one. These two image portions have the same luminance and give rise to the same corresponding neural activity level. What is it, according to the nonisomorphistic approach, that makes one portion look brighter than the other? It is clearly not the local luminance or neural activity level. Plausibly, the global brightness difference depends, in some way, on the asymmetrical distribution of local neural activity corresponding to the common edge of the two rectangles; but what is the causal machinery that supports and explains this dependence? The step-to-cusp transformation from Figure 1a into Figure 1c can be explained using mechanistic concepts such as receptive field shape and sensitivity profile, lateral interactions, or bandpass channels. In contrast, the mathematically complementary cusp-to-step transformation from Figure 1c into Figure 1e is merely asserted to take place, but is apparently left without any mechanistic explanatory basis.

One interpretation of the nonisomorphistic approach, noted by a referee, is that it only points out that to explain the COCE there is no logical requirement that a specific neural action, such as a filling-in process, should occur after the formation of the neural cusp. As an alternative, one could propose that the brightness percept can be accounted for by subsequent symbol-based or informationbased processes. This idea is an instance of the "cognitive" approach to the explanation of the COCE discussed in Part 3. Note, however, that such subsequent processes most likely would have some physiological basis in the brain of the perceiver. In consequence, in this interpretation the nonisomorphistic approach would lose much of its initial explanatory appeal. First, it would no longer be true that the neural cusp was the sought-for end process that underlies appearance. Something else would also have to go on in the nervous system, an interpretative process following the formation of the cusp, for the appropriate percept of the brightness step to be generated. Second, the nonisomorphistic approach would cease to be more parsimonious than the alternative approaches, since it too would have to include additional processing stages that would need to be specified and tested.

Another reading of the nonisomorphistic approach, which may be closer to what its proponents have in mind, is that there simply are no additional processing stages, and that a neural cusp is, at the physiological level, all that is needed for a brightness step to be perceived. Now, 
as noted in Part 1, the properties of retinal neurons suggest that, given a luminance step or cusp as a stimulus, a neural cusp is already formed at the level of retinal ganglion on-cells. However, the retina is an unlikely bridge locus. Most visual scientists would probably be more inclined to assume that cortical, rather than retinal, tissue was the more immediate substrate of percepts. Consequently, if one wants to maintain the idea that neural cusps underlie brightness steps, one could assume that the cuspshaped retinal distribution is preserved in the course of subsequent cortical processing, at least to the extent that its shape reappears at the final neural stage giving rise to the percept. In other words, to be consistent, the nonisomorphistic approach should assume that an isomorphic (or at least approximately shape preserving) map occurs between the retinal distribution and the bridge locus. Such an assumption of the preservation of attributes of early processing levels at later stages is an instance of Teller's (1980) "and nothing mucks it up" " proviso, shared by any theory that assumes that properties of relatively peripheral neurons explain properties of percepts.

If this is granted, it follows that the nonisomorphistic and the isomorphistic approaches to the COCE are, at least formally, much more similar than they initially might have appeared to be. Both approaches have to account for how a luminance cusp distribution is transformed into a perceptual step distribution. Both assume an intermediate early neural cusp distribution, but disagree as to whether the final neural distribution is a cusp or a step. The nonisomorphistic approach assumes an isomorphic cuspto-cusp mapping from the early neural representation into the bridge locus, followed by a nonisomorphic cusp-tostep mapping from the bridge locus into the percept. In the isomorphistic approach, it is the other way around: the retinal cusp is first nonisomorphically transformed into a cortical step, which is then isomorphically mapped into the perceptual step. Note that the labels for the two approaches were chosen according to the type of the presumed final neural-perceptual map.

From the formal point of view, both approaches assume essentially the same two types of maps, executed in a different order. However, from the explanatory point of view, the order chosen in the isomorphistic approach may be more advantageous. The main challenge for both approaches is to account for the nonisomorphic map. In the isomorphistic approach, the material substrate of this map is the visual neural system. There are currently no physiological data providing direct support for such a map. However, it is a testable proposition, and there is no lack of potential neural candidates to implement it: it is known that visual processing involves a number of cortical areas, each composed of several layers containing a variety of neuron types that differ in processing characteristics and interconnection structure (van Essen, 1985). In contrast, in the nonisomorphistic approach, the nonisomorphic map does not belong to the neural domain, but represents a relationship between neural and perceptual distributions. However, as noted earlier, it is not at all clear how such relationships are implemented; nor is it clear what the constraints on them are or how different proposals should be tested and evaluated. These difficulties are not surprising, because this map is an aspect of the notorious mindbody problem. The isomorphistic approach cannot avoid this problem either. However, conceptually, the idea of an isomorphism between certain aspects of neural activity and certain aspects of percepts may be more acceptable, at least within a general reductive stance that assumes that, at some level of description, perceptual states are neural states.

A shortcoming of the preceding analyses is the probably oversimplified notion of the bridge locus. Both approaches, as presented here, assume that neurons of a single type are responsible for the brightness percept. However, visual cells sensitive to luminance and luminance contrast may differ on many other dimensions. They include differences in receptive field sizes and shapes, as well as differences between ON versus OFF, simple versus complex, transient versus sustained, and broadband versus color-opponent neurons. It is difficult to assess the extent to which a given neuron class contributes to the brightness percept, but there is no compelling reason to believe that the bridge locus is confined to neurons of a single type within a single cortical visual area. As an alternative, it is conceivable that the brightness level of a small portion of the visual field depends on the activity of many different cell types at corresponding loci in several topographically organized and interconnected areas. Nevertheless, given the lack of relevant data, the single-cell type of bridge locus is a heuristically useful assumption.

\section{Demonstrations}

Most studies of the COCE have used either displays similar to Plate 1 or circularly symmetric versions created by spinning painted disks (Cornsweet, 1970). The structure of the luminance variation in such images is essentially one-dimensional. In this section, several displays that contain luminance cusps will be presented, but these have a more complex structure and can therefore not be adequately represented with a single one-dimensional luminance distribution such as that of Figure $1 b$. The displays demonstrate that, depending on the conditions in the surround, the same luminance cusp may or may not give rise to the COCE. They thus raise difficulties for the nonisomorphistic approach by illustrating its insensitivity to the effects of visual context.

The first example concerns the effect of the luminance of the surround. In Plate 1, this luminance is lower than the average luminance of the two rectangular regions. If this surround is replaced by one in which the luminance is higher than the average luminance of the rectangles, the perceptual consequence is that the average brightness of the two central rectangles decreases, due to brightness contrast, but the difference in brightness between two regions remains, that is, the COCE. However, the percept changes dramatically with a surround whose lumi- 
nance is equal to the average luminance of the rectangular regions. This effect is demonstrated in Plate 2 (from Todorović, 1983).

The transformation of Plate 1 into Plate 2 can be enjoyed in our laboratory with the help of a computer program that gradually increases the luminance level of the surround, leaving the luminance profile of the two rectangular regions unchanged. During most of this transformation, a difference in brightness between the two rectangles can be observed. However, when the surround luminance reaches the average level of the central luminance, the two rectangles disappear, and the COCE is abolished. The isolated vertical brightness cusp in Plate 2 corresponds approximately to the physically real luminance cusp. Displays containing more than one isolated luminance cusp also fail to evoke the COCE (Arend \& Goldstein, 1987). Unlike Plate 1, in Plate 2 there is no convincing brightness difference of portions of the display that are removed from the location of the luminance cusp. The important thing to note is that Plate 1 and Plate 2 contain identical luminance cusps, so that they are likely to induce equivalent neural cusp distributions. However, only Plate 1 involves a clear brightness step distribution, that is, the COCE. Thus, a rule that only states that a neural cusp corresponds to a brightness step is inadequate; a more complete account of the COCE must involve more than the analysis of the neural effects of luminance cusps.

One way to describe the difference between Plates 1 and 2 is that in Plate 1, but not in Plate 2, the luminance cusp coincides with a boundary between two regions. Thus, one way to construct a more complete theory of the COCE might be to include topological constraints in its formulation. For example, one could propose a modified theory in which luminance cusps caused a COCE only if they formed parts of boundaries of topologically closed regions. The physiological basis for such a rule would remain to be established. I will now discuss several visual situations to argue that the inclusion of topological criteria is neither straightforward nor sufficient to fully explain the phenomena.

First, note that one way to induce a COCE in Plate 2 is to prepare a rectangular frame with dimensions and color of the dark background in Plate 1. If such a frame is superposed in the appropriate way onto Plate 2 , the luminance conditions of Plate 1 will be recreated, and the COCE will appear. Imagine now that the size of the central hole of this frame gradually increases in both dimensions. In this way, as the dark frame grows increasingly thinner, Plate 1 can be transformed into Plate 2. When such a transformation is performed on a monitor, one observes that the manner of perceived change of topological structure does not completely correlate with the way brightness is transformed. Even a small increase in the size of the hole changes the topological structure of the display, in that the vertical luminance cusp no more forms a boundary between two regions. However, although the change of the perceived topological structure is relatively abrupt, the change of the brightness percept is not. The COCE disappears only gradually with the increase in size of the hole, and is still present even when the endpoints of the cusp are clearly perceived not to reach the horizontal inner sides of the frame.

A second display of interest for the study of the interplay of topology and luminance in the determination of brightness is provided in Plate 3. It contains two vertical luminance cusps, one directly above the other, whose luminance profiles are mirror images. Note that the presumed topological condition for the appearance of the COCE is satisfied: the cusps form parts of region boundaries. Indeed, if one occludes the upper half of the display, a COCE appears in the lower half; if one occludes the lower half, a COCE of reversed polarity appears in the upper half. However, the appearance of the whole, unoccluded display does not quite seem to be the sum of the appearances of its two halves. On the basis of the effects in the occluded displays, one might have predicted an abrupt brightness difference between the upper and the lower quadrants in the left and right halves of the unoccluded display. However, the actual distribution of brightness is much more gradual. There is no horizontal brightness step dividing the display into a lower and upper half. Note that the conspicuous brightness differences across the vertical symmetry axis and horizontally, at the center of the display, correspond to physically real luminance differences induced by the cusps, and are not illusory.

Plate 4 is a further development of the construction used in Plate 3. It contains four columns consisting of vertical concatenations of luminance cusps of opposite polarity. In contrast to Plate 3, in Plate 4 illusory horizontal lines may be noted, separating regions of illusory unequal brightness. These effects are somewhat unstable, and may be enhanced with prolonged fixation. The appearance of this illusory chessboard may be compared with subjective contour figures (Kanizsa, 1979) that also involve illusory lines and brightness effects. The difference between the effects in Plate 3 and Plate 4 may be accounted for if one assumes that the process that generates the illusory lines needs at least two spatially separated induction loci. In Plate 4 , such inductors are provided by pairs of loci of cusp polarity reversal situated in neighboring columns at the same vertical height. Related explanations of phenomena involving subjective contours were described by Shapley and Gordon (1985) and Grossberg and Mingolla (1985a, 1985b).

Finally, another topologically interesting display is presented in Plate 5. All brightness steps in this display are due to straightforward luminance steps, except for the short vertical edge in the lower central portion of the display, which is caused by a luminance cusp. The rectangular "annulus" is a topologically connected visual region, meaning that any two points belonging to that region can be connected by a (not necessarily straight) line contained wholly within the region, without crossing any brightness step. The peculiar fact about this region is that it contains an edge, the one caused by the luminance 
cusp, both sides of which belong to the same region. The lesson here is that a brightness step that forms a part of a boundary of a region is not necessarily a border between two different regions.

To summarize this part, two types of difficulties are raised for the nonisomorphistic theory. The first concerns the consequences of the hypothesis that neural cusps can underlie brightness steps. It is claimed that this theory is incompletely specified, that it fails to provide the mechanism that underlies the brightness effect, and that it offers no gain in parsimony over competing explanations. The second type of difficulty involves the contextual sensitivity of the COCE, demonstrated by several displays. They present difficulties for any theory of this effect that restricts its scope to effects of luminance cusps. It is also argued that the inclusion of topological criteria does not provide the appropriate constraints for an adequate explanation of the effects in the displays.

\section{PART 3 THE COGNITIVE APPROACH}

The foregoing discussion implies that any theory of the COCE that strives to explain the structure of the appearance of the phenomenon should involve processing stages that go beyond an early retinal distribution of neural activity. In Fourier analysis terms, the attenuation of lowfrequency components should be followed by their partial regeneration. The nature of these additional processes is discussed in a number of papers. The theories can be subdivided in two groups, a dichotomy familiar to perception scientists. Theories in the first group, discussed in this part of the paper, invoke cognitive, interpretative, strategic, or intelligence-like processes. Theories in the second group, discussed in Part 4, rely on noncognitive, mechanistic, computational, or physiologically based processes. The two approaches are not logically exclusive, since one would expect that cognitive processes should ultimately be founded on physiological mechanisms. However, there is a clear difference in emphasis and explanatory apparatus.

Currently there exists no well-developed cognitively oriented theory of the COCE. However, several authors have suggested that cognitive factors may play a role in this phenomenon. For example, van den Brink and Keemink (1976) noted that some figures involving shallow luminance gradients contain areas whose brightness depends on the perceived three-dimensional structure of the display. They concluded that "the source of this illusion may be situated at a much higher level, where neural excitation patterns are recognized and interpreted" (p. 155). However, perceptual multistability does not necessarily imply "higher" processes. A slight cognitive flavor can also be detected in papers by Tolhurst (1972) and Shapley and Tolhurst (1973), who proposed that luminance cusps were detected by appropriately located and oriented cortical simple cells, and that "any pattern which excited this set of neurons would be interpreted as a sharp contour dividing two areas of unequal brightness"' (Shapley \& Tolhurst, 1973, p. 166). Such an interpretative process could perhaps explain why the COCE is present in Plate 1 , but could not explain, at least not without further elaboration, why it is absent from Plate 2. Coren's (1983) study of the COCE, described later in this paper, led him to the conclusion that strategic factors decide whether the percept will be dominated by absolute or by relative brightness information. However, Cohen and Grossberg (1984) offered an explanation of the same data without relying on cognitive processes.

Finally, Dooley and Greenfield (1977), referring to luminance cusps as "Cornsweet edges," noted that "a lowcontrast real step results in a signal within the visual system much like that due to a Cornsweet edge. The visual system interprets them both as steps because both the real step and the Cornsweet edge have the same general profile and because a step is the most usual signal that has such a profile"' (p. 764). There are logical problems with this explanation. A luminance step may, indeed, be more usual than a luminance cusp, or any other member of the "equivalence set" of distributions described by Ratliff and Sirovich (1978), all of which cause percepts of brightness steps. But they all presumably have the same effect on the visual system; so how could perceivers detect the difference in their frequency of occurrence? How could they come to know the shape of the luminance profile of the "most usual" member of this set, so that they could subsequently base their interpretations of neural cusps on that shape?

In sum, it seems fair to conclude that cognitively oriented explanations of the COCE have not, at least not as yet, made a significant contribution, and that it still remains to be established that such an approach to this effect is necessary or fruitful. Indeed, the COCE may be as good an example as any of a "cognitively impenetrable" phenomenon. We know, cognitively, that apart from the gradients, the two rectangles in Plate 1 are equiluminant. Our nervous systems "know" it at an early processing stage where the two parts of the stimulus evoke equal activity levels. Moreover, as noted in Part 1, the equality can easily and repeatedly be perceptually confirmed by the simple operation of border occlusion. However, despite all of this information, as soon as the occlusion is removed, the brightness difference is restored.

\section{PART 4 THE MECHANISTIC APPROACH}

Most explanations discussed so far refer specifically to the COCE. In contrast, most explanations that will be discussed in the following sections have a broader scope. They try to account for a variety of brightness perception phenomena, and deal with the COCE as a specific test case. These theories generally share the assumption of an early edge-enhancing stage. Following this stage, 


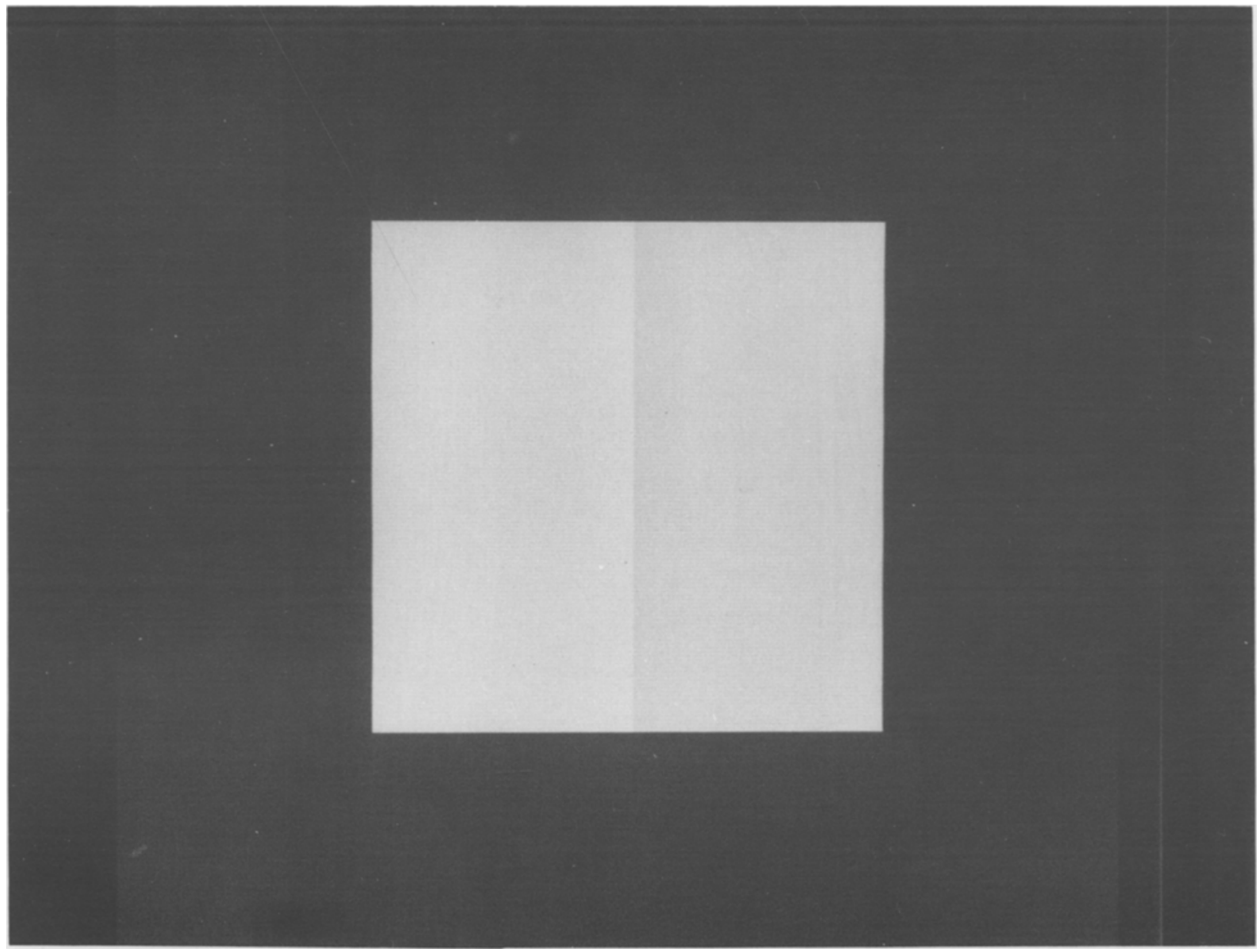

Plate 1. A standard version of the COCE. Figure 1b depicts qualitatively the luminance distribution of a horizontal cross-section of the central portion of this display. The luminance of the two rectangles is identical, except for a narrow region at their common border, which contains a positive luminance gradient in the left rectangle, and a negative luminance gradient in the right one. The perceptual consequence is that the left rectangle looks uniformly brighter than the right one. The approximate brightness distribution is depicted in Figure 1f. 


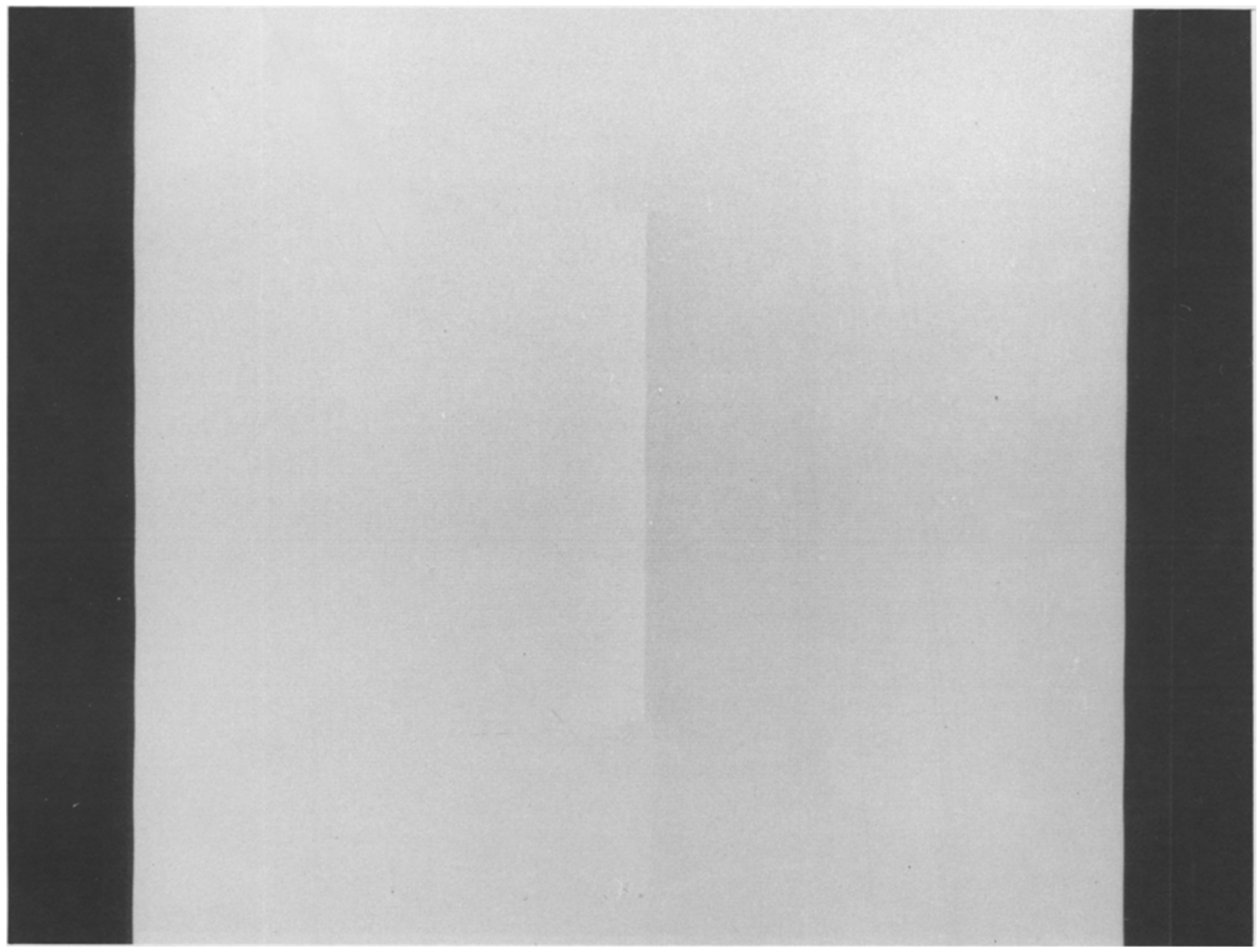

Plate 2. A luminance cusp embedded into a uniform background. The luminance distribution of the cusp is identical to the one in Plate 1 , but without the contrasting background, no COCE appears. 


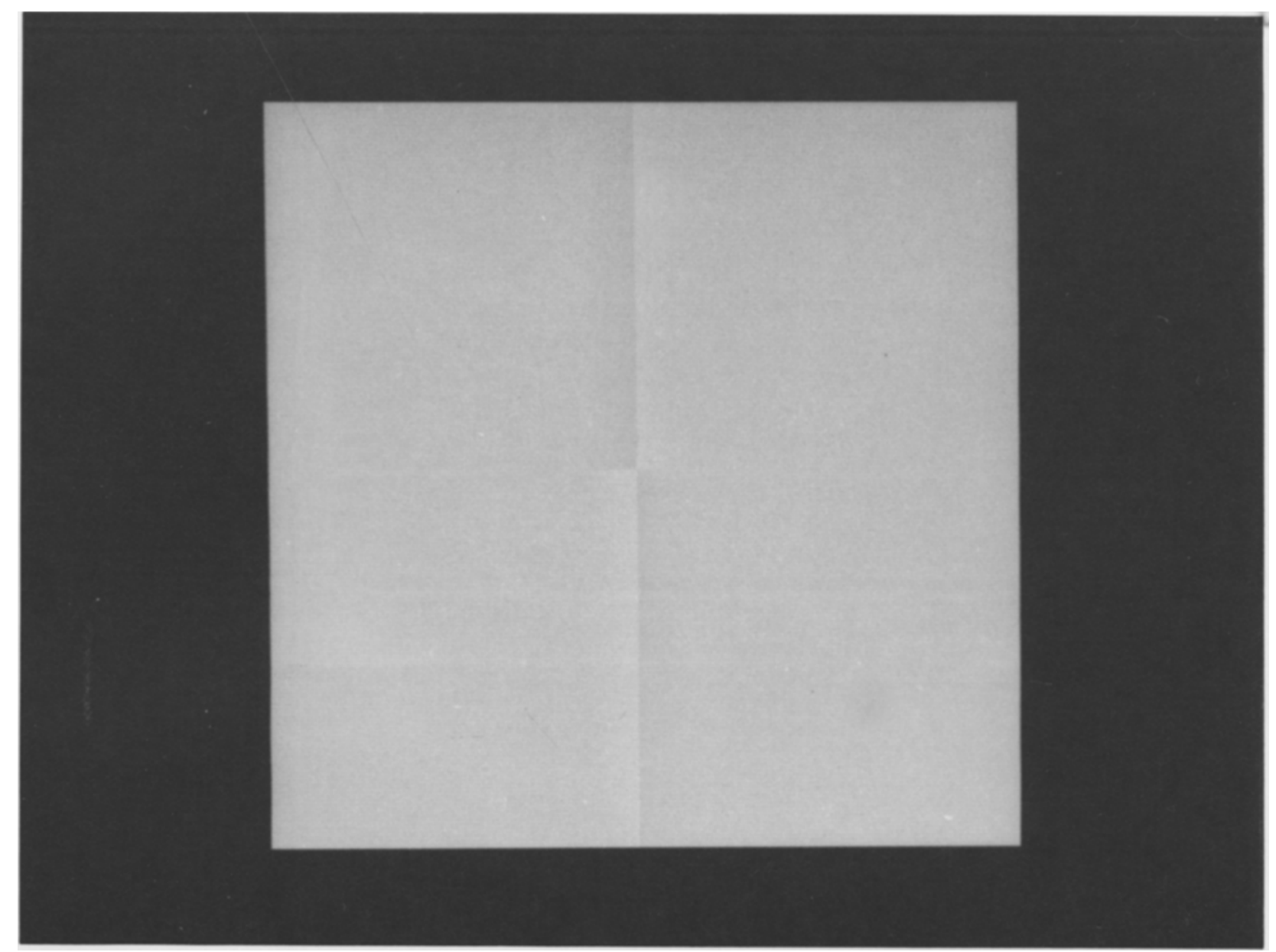

Plate 3. Two luminance cusps of reverse polarity in vertical alignment. The horizontal luminance profile of the lower half of the picture is similar to Figure 1b. The luminance profile of the upper half is its mirror image. Occlusion of either half induces a COCE in the other. However, in the unoccluded display there is no abrupt step between upper and lower quadrants. 

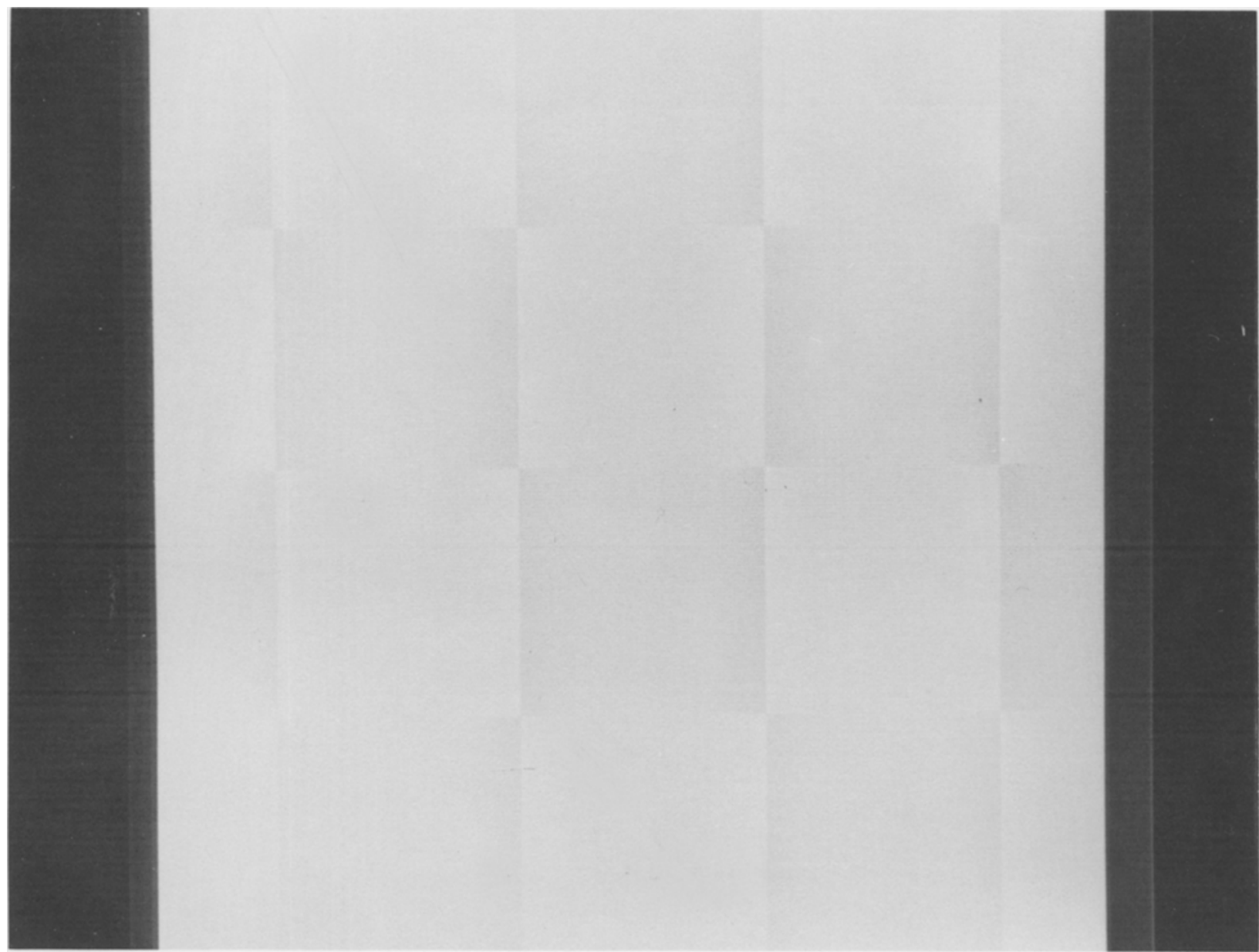

Plate 4. An elaboration of the visual situation in Plate 3. This figure contains 16 vertical luminance cusps, 8 of each polarity, arranged in four vertical columns. Each cusp has opposite polarity from its nearest horizontal or vertical neighbor. The perceptual consequence is a partly illusory chessboard. The horizontal spatial extent of each luminance gradient is approximately equal to one-fifth of the distance between the midline of two columns. Thus, the vertical central three-fifths of the areas of each "white" or "black" field have the same luminance. 


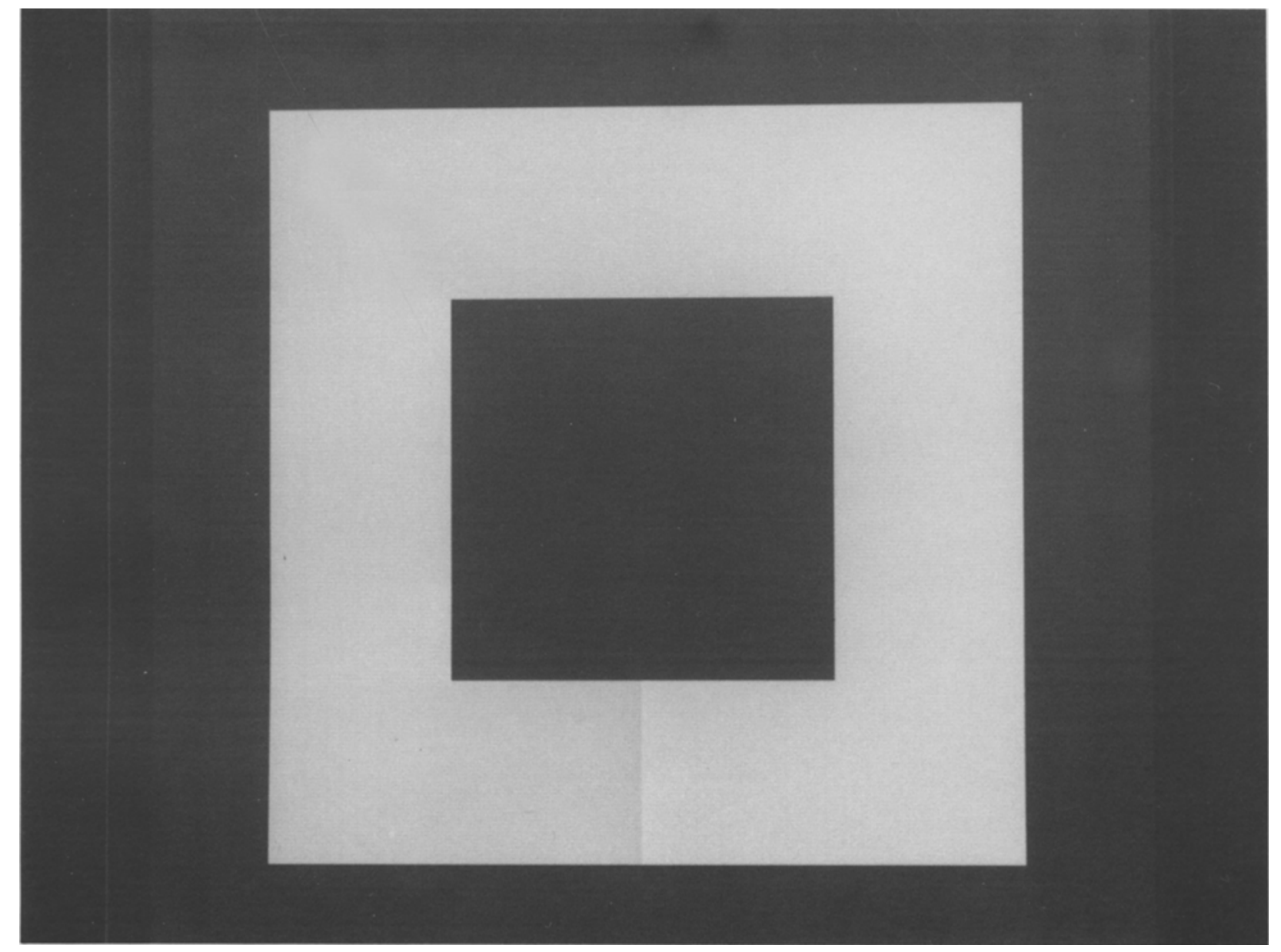

Plate 5. A short vertical luminance cusp is embedded in the middle of the bottom side of an otherwise uniform rectangular annulus. See text for details. 


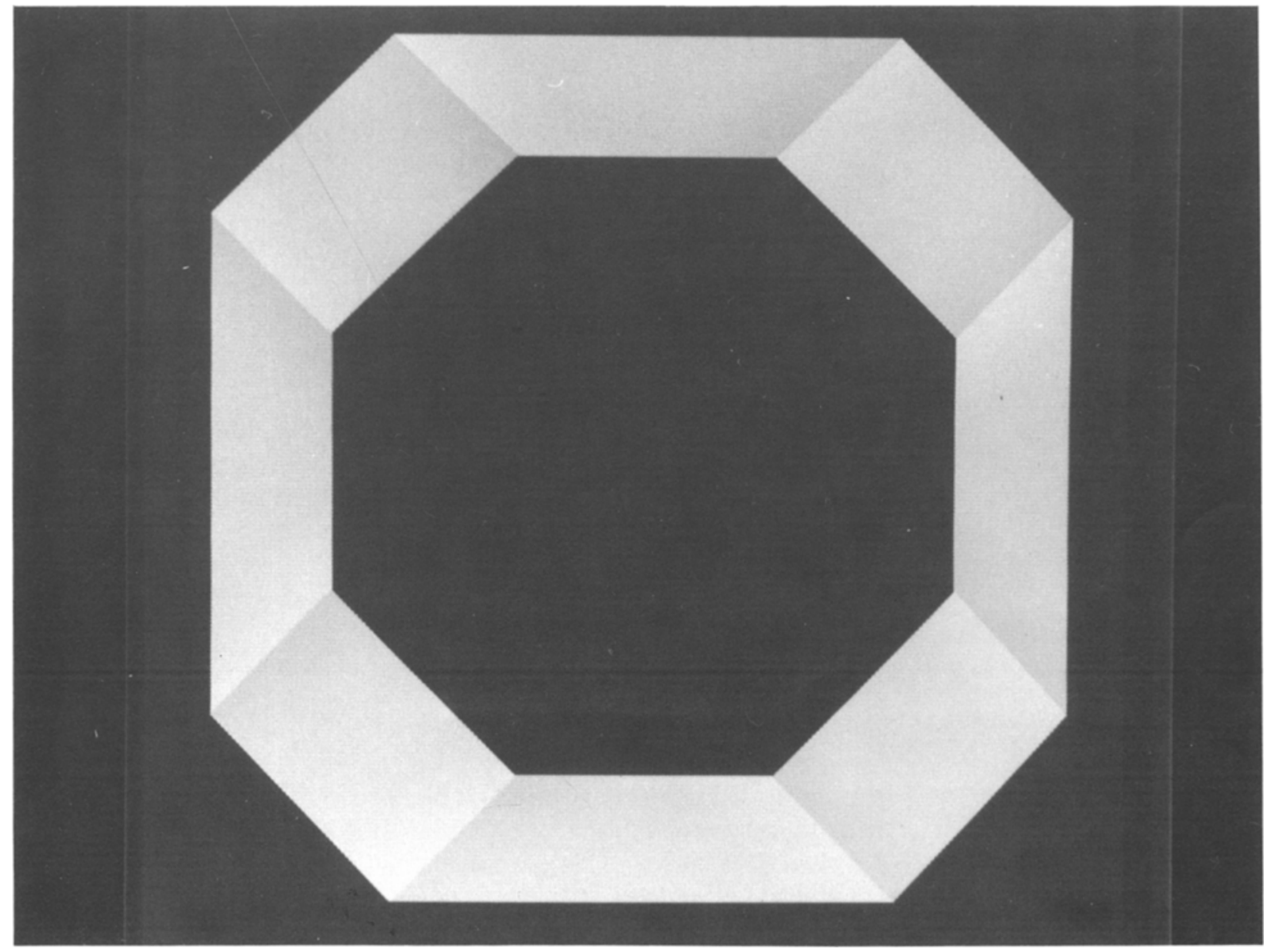

Pate 6. An "impossibie" brightness staircase. Each of the eight regions of the octagonal "annulus" has the same luminance distribution, with a positive brightness gradient at one edge balanced by an equivalent negative brightness gradient at the other edge, and a homogeneous middle portion. The display should be occluded such that only two or three neighboring regions are in view. Each region then assumes a brightness level midway between its immediate neighbors at each side. The effect is most convincing when the photograph is viewed from a short distance. In the unoccluded display, the brightness relationships between regions are more ambiguous. 


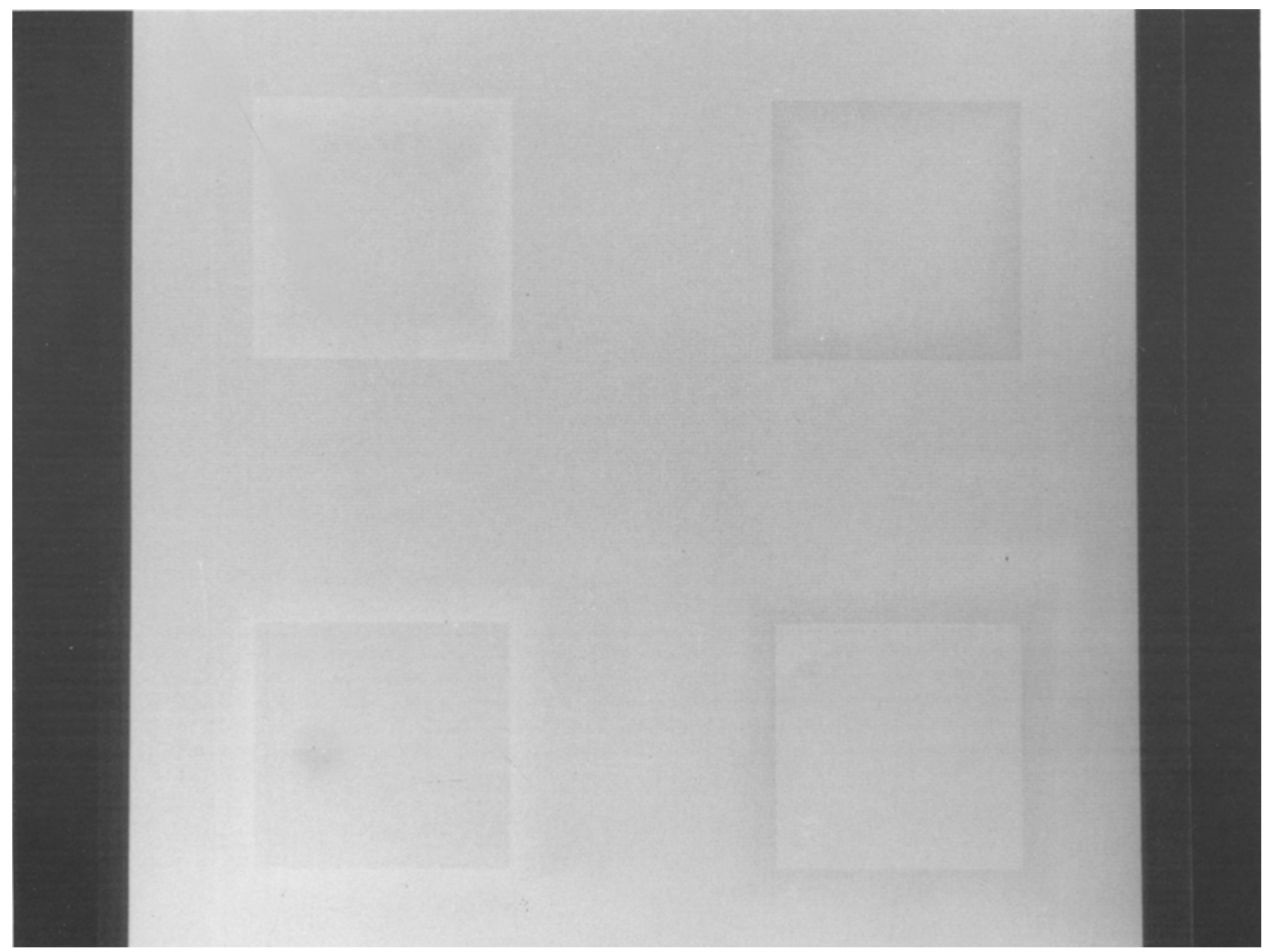

Plate 7. The effects of four types of luminance gradients on region brightness. The luminance cross-sections of the four regions are presented in Figure 3. In this display, only the outer luminance gradients (in the bottom two regions) have an immediate, perceptually salient influence on the brightness of region interiors. Steady fixation of either of the top regions may, with some effort, induce a "fillingin" effect. See text for details (figures $a, b, c$, and $d$ correspond to figures $a, b, c$, and $d$ in Figure 3 ). 


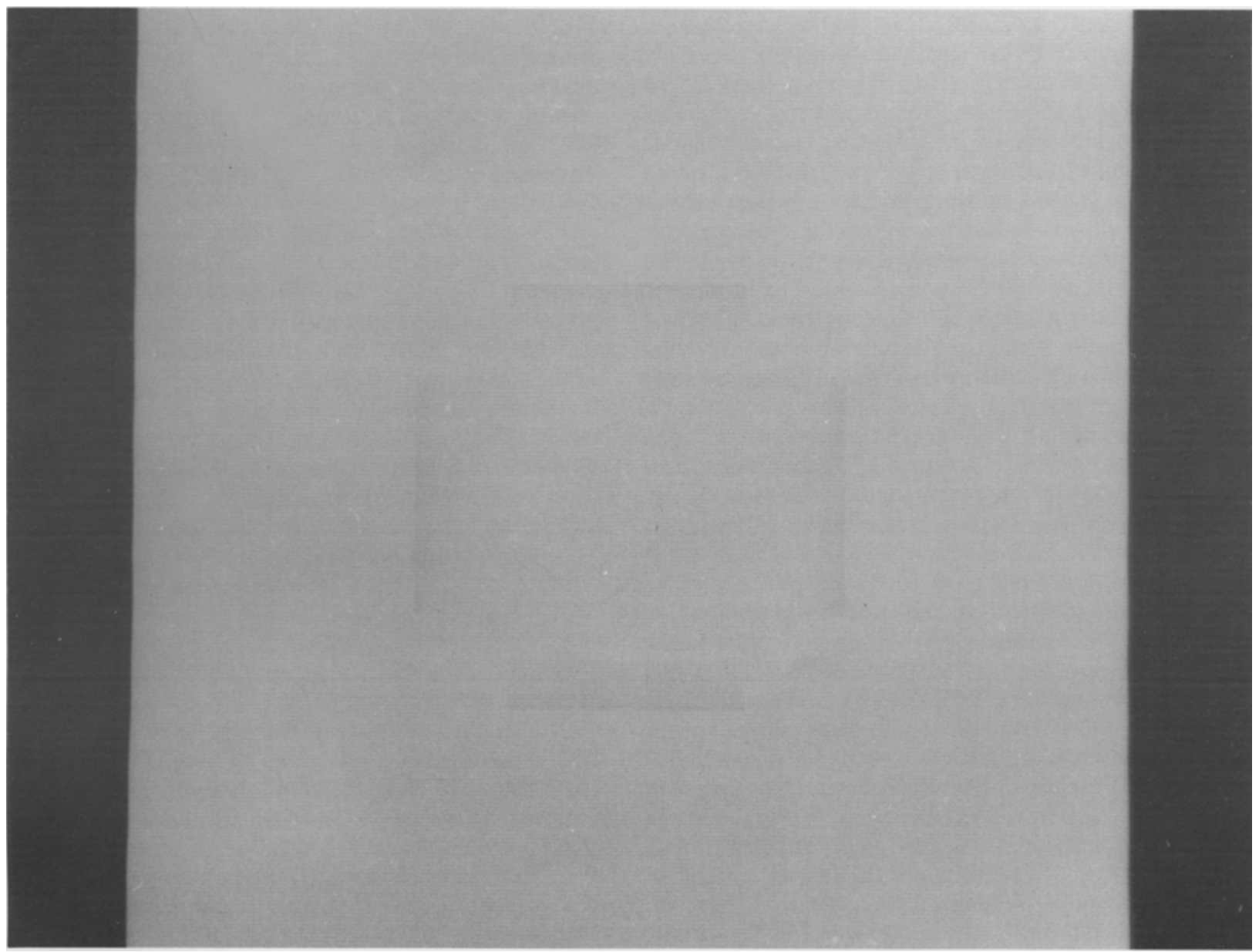

Plate 8. The influence of stimulus motion on filling-in. See text for description and instructions. 
subsequent processing stages are postulated, resulting eventually in an activity profile approximately isomorphic with the brightness distribution.

Theories within this approach can be tentatively classified in two groups. Those in the first group emphasize the description of visual processing in terms of standard mathematical operations, such as differentiation and integration (Arend, 1973, 1985; Arend \& Goldstein, 1987; Blake, 1985; Davidson \& Whiteside, 1971; Horn, 1974, 1986; Schiffman \& Crovitz, 1972). Theories in the second group use a more physiologically oriented framework, invoking the dynamics of neural processing at different levels of the visual system (Cohen \& Grossberg, 1984; Hamada, 1985; Ratliff, 1971, 1972). Earlier related theories, which do not refer specifically to the COCE, were presented by Fry (1948), Gerrits and Vendrick (1970), and Walls (1954). These two groups of theories are termed here "integration theories" and "filling-in" theories, respectively, according to the postulated mechanism that is crucial for the explanation of the COCE. Although there is a clear difference in emphasis, the two approaches are far from incompatible, especially in computationally oriented theories: some filling-in theories involve mathematically specified transformations, related to differentiation and integration, and some integration theories use neural-like models involving computational units whose processing architecture and interconnectivity implement the mathematical operations.

\section{Integration Theories}

Integration theories assume that the visual processing stages involved in brightness perception, and specifically in the COCE, may be modeled by mathematical operations of differentiation and integration. The derivative of a function is another function, representing the rate of change of the original function; thus, slow changes of the original result in near-zero values of the derivative, and abrupt changes produce sharp peaks and troughs. This transformation is analogous to the early edge-enhancing stage of visual processing. On the other hand, integrating the derivative essentially restores the original; this property makes integration appealing as a model for the recovery of the shape of the luminance distribution.

However, the COCE cannot be explained by simply following up derivation by integration. This is because the derivative of a cusp and the derivative of a step are not mathematically equivalent. Exact integration would restore the shapes of the step and the cusp from their respective derivatives. One solution is to interpose a thresholding stage between the two operations. Only the effects of relatively large, abrupt luminance steps survive this stage, whereas the effects of small, subthreshold ones, such as shallow luminance gradients, are reduced to zero. The thresholded derivative of a cusp is similar to the derivative of a step, which the integration procedure then "restores," thus generating the COCE.

Although the basic differentiation-thresholdingintegration sequence is common to most integration the- ories, there are also differences. They include the order of integration and the dimensionality of the luminance distributions. For example, Arend (1973) used first-order derivatives and one-dimensionally varying luminance distributions, Schiffman and Crovitz (1972) used onedimensional second-order derivatives, Blake (1985) used two-dimensional first-order derivatives (magnitudes of gradients), and Horn $(1974,1986)$ used two-dimensional second-order derivatives (Laplacians). These differences are important for several reasons. First, roughly speaking, increasing order of differentiation and dimensionality tends to increase the amount of additional mathematical information necessary to recover the original. In particular, the differentiation step involves loss of the absolute luminance-level information. Second, although the two-dimensional theories are mathematically more complex, such an approach is essential for a more complete account of the phenomenon. This is clearly shown by several demonstrations in this paper. A model that deals only with one-dimensionally varying luminance distributions cannot distinguish between Plates 1 and 2, and cannot adequately handle Plates $3,4,5$, and 6 . Finally, the choice of the derivative is relevant for the physiological reality of a model. The rotationally symmetrical Laplacian can serve as an approximation for concentric antagonistic receptive fields, whereas directional derivatives are better models for some types of cortical simple cells.

The rest of this section is devoted to a discussion of two problematic features of integration theories. The first such feature is most explicitly expressed in Arend's (1973) paper. It is the prediction that abrupt luminance changes can influence the brightness levels of distant regions across several intervening borders. This assumption explains the brightness levels of several complex luminance profiles presented in Arend, Buehler, and Lockhead (1971), some of which contain both luminance steps and cusps. However, there are other luminance profiles that present difficulties for integration theories.

For an illustration of this aspect of the theory, consider first the luminance profile depicted in Figure 2a. The distribution is homogeneous, except for the negative luminance gradient at one end, balanced by an equivalent positive luminance gradient at the other end. Figure $2 c$ depicts a shallow linear gradient. Profiles such as Figures $2 a$ and $2 \mathrm{c}$ can be used as building blocks for more complex profiles. For example, Figure $2 b$ depicts a luminance profile constructed as the conjunction of two profiles of the type $2 \mathrm{a}$; note the similarity to Figure $1 \mathrm{~b}$. The appearance of such a profile, embedded in a dark surround (not presented in this paper), is very similar to Plate 1 . The left region is brighter than the right one, and the luminance gradients at the nonshared borders are hardly noticeable. Analogous brightness effects are induced by the distribution in Figure 2d, the conjunction of two profiles of type 2c (Coren, 1983; Land \& McCann, 1971; van den Brink \& Keemink, 1976). Thus, for such effects to appear, the exact shape of the luminance distribution is of less importance than the condition that an abrupt luminance step be flanked by shallow luminance gradients. 


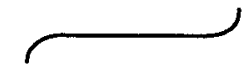

(a)

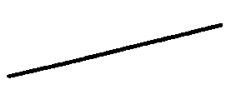

(c)

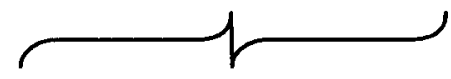

(b)

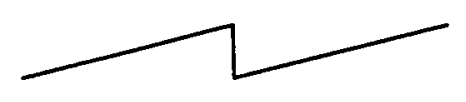

(d)
Figure 2. Simple luminance distributions as building blocks for more complex ones. (a) A luminance profile in which the negative luminance gradient at one end is balanced by the positive luminance gradient at the other end. (b) The conjunction of two profiles of type a. (c) A shallow linear gradient. (d) The conjunction of two profiles of type $c$. Note that in both $b$ and $d$ the left and right halves of the profile have an identical average luminance, but the left half would look brighter than the right one.

Consider now a profile consisting of a linear conjunction of more than two simple profiles. Arend's (1973) theory predicts that, for any number of such regions, a brightness staircase should be perceived, with each succeeding region being darker than the previous one. The reason for this is that the integration process transforms each abrupt luminance change into a brightness step. Thus, with each additional region, the brightness difference between the first and the last member of the chain should cumulatively increase. Arend et al.'s (1971) study confirms this prediction for a series of three concentric regions of a bull's-eye with cusp-shaped borders. However, data by Coren (1983), who studied conjunctions of gradients of type $2 c$, disconfirm the prediction. Although two gradients induce a COCE, the appearance profile of the conjunction of five gradients is relatively flat, showing no cumulative increase in brightness. This experiment was not reported in much detail, and more parametric studies are needed of the scope of cumulative brightness increase over several luminance cusps. Still, the result presents a serious difficulty for integration theories.

A unique challenge for the notion of perfect cumulativeness is created by the transformation of the openended, linear chain of regions into a closed, circular one. Such an example is presented in Plate 6. This is an elaboration of a display from Todorović (1983). Arend and Goldstein (1987) have studied a variant of such a display. A related display, not involving the COCE, was presented by Ullman (1984). Plate 6 consists of eight regions forming an octagonal "annulus" on a dark background. The luminance distribution along the longer axis of each region is similar to the one in Figure 2a. Thus the luminance profile taken perpendicularly across each short, shared edge is cusp shaped. Note that Plate 5 is a degenerate version of the idea of closure, with only one cusp and one connected homogeneous region.

As was the case with some previous displays, portions of Plate 6 appear different depending on whether some other portions of the display are occluded or not. On the one hand, if one occludes most of the display, such that only two or three component regions can be seen in entirety, a relatively convincing COCE appears. Under such conditions, each region in the display looks darker than its neighbor in the clockwise direction and brighter than its counterclockwise neighbor. On the other hand, if the observer inspects the whole picture, without long fixations, he or she will have quite a hard time consistently deciding which region is brighter or darker. Under these conditions, the regions become less uniform in brightness, and the gradients become more prominent. However, if one fixates one's gaze and attention on only one region or edge in a close-up view, a COCE will appear, similar to the one in the occluded display. Most of these observations have been experimentally confirmed by Arend and Goldstein (1987), who studied a four-sector circular figure with radial profiles of type $2 \mathrm{c}$.

This display has a paradoxical aspect, reminiscent of the "impossible staircase" of Penrose and Penrose (1958) and Escher (1961), and of the "circular pitches" of Shepard (1964). The brightness relations of neighboring regions are locally consistently transitive, but their circular arrangement induces a global inconsistency. The conflicting percepts have an analogy in the appearance of the impossible staircase, in which the perceived spatial relations of a small number of neighboring steps are unambiguous, but a consistent three-dimensional structure of the whole drawing is impossible to achieve.

A straightforward extension of one-dimensional integration would have trouble explaining these appearances. Consider an integration process, starting at any short, shared edge, proceeding in the clockwise direction through all eight regions, and finishing at the other side of the starting edge. Call the first region traversed by this process $A$, and the last one $B$; B is the counterclockwise neighbor of $A$. The integration process would predict that each succeeding region in the clockwise direction would be brighter than the preceding one, thus making $B$ much brighter than $\mathrm{A}$. However, the brightness difference in the unoccluded display is, if anything, in the other direction. In addition, this wrong prediction of a large brightness contrast applies to only one edge, the one that happens to be the starting and ending place of the integration process. However, the contrasts across each edge in Plate 6 look similar, with no edge having a distinguished appearance. Finally, if the integration process should happen to start within the interior of a region then, after the conclusion of this process, either a brightness edge should appear within that homogeneously luminant region, which does not happen, or a region should be perceived as brighter than itself, which is unlikely to happen.

The second problematic feature of integration theories will be illustrated with a prediction based on Horn's (1974, 1986) theory. He presented continuous and discrete, oneand two-dimensional versions of a mathematically specified procedure designed to correctly recover surface reflectances in the face of continuous luminance variations 
induced by light sources. The two-dimensional luminance distribution is transformed using Laplacians as differentiation operators, and the resulting distribution is thresholded. The integration step is formulated as the solution of a partial differential equation of the Poisson type, using the method of Green's function. The thresholding stage, whose function is to suppress illumination gradients, was modeled after Land's retinex theory (Land, 1977, 1986; Land \& McCann, 1971). An implementation of the mathematical operations in a multilayered hierarchy of computational units was also described. Blake (1985) presented an improved version of Horn's model, involving additional mathematical analyses and changes in its internal structure. A related analysis was offered by Arend (1985) and Arend and Goldstein (1987). Terzopoulos (1986) provided yet another, more efficient computational scheme, involving image processing at several scales simultaneously. Frisby (1979) described a possible neural implementation based on Horn's model. Hurlbert (1986) presented a review of several computation-' ally oriented brightness theories, and showed them to be special cases of a general mathematical formulation.

Horn (1974), Frisby (1979), and Blake (1985) presented a simulation of one of the standard versions of the COCE. Horn (1974) also included a demonstration (Figure 11 in his paper), similar to Plate 2 in this paper, involving a luminance cusp embedded in a uniform background. The difference is that in Horn's figure the shape of the contour is not straight, as in Plate 2, but semicircular. The output of the simulation was presented but not discussed in any detail; it appears that the theory predicts far-ranging spreading of the influence of the edge contrast, as in the standard COCE, but contrary to the appearance of Plate 2.

The problematic feature of Horn's algorithm, when compared with human visual processing, is its exclusive orientation on reflectance recovery. This feature helps to explain brightness constancy, but is troublesome for explanations of simultaneous brightness contrast. Consider the standard form of this effect: a gray patch on a dark surround looks brighter than an equiluminant patch on a bright surround. This visual situation contains neither reflectance gradients nor illumination gradients. Thus, the thresholding stage of integration theories has no gradient effects to threshold, no nonlinearities are introduced, and the relative output values mirror the relative input values. Consequently, equiluminant surfaces are predicted to appear equally bright. Horn (1974) did note that a simple modification in the differentiation stage should make the output of the theory "suffer from Mach bands and simultaneous contrast effects," but this claim was not supported by appropriate simulations.

Theories of the COCE should pay attention to brightness contrast effects for two reasons. First, these effects are strong, and any brightness theory that does not handle them is incomplete. The change of surround luminance can change the appearance of the surrounded area from "very bright" to "very dark" (DeValois, Webster, \& DeValois, 1986; Heinemann, 1972; Hering, 1878/1964;
Wallach, 1948; Yund \& Armington, 1975). The occasional claim that brightness contrast and the Mach bands can be explained by the same mechanism of lateral inhibition is doubtful. Brightness contrast differs from Mach bands in that it spans much larger extents of visual space, it is not dependent on ramp-like changes of luminance, and it does not visibly diminish with distance. In addition, recording from the lateral geniculate nucleus, DeValois and Pease (1971) found evidence for neural correlates of Mach bands but not for brightness contrast, suggesting that the latter effect is cortical in origin.

The second reason for the importance of brightness contrast is that it appears that this effect and the COCE are closely related. Support for this claim is presented in Plate 7. The stimuli in this figure contain single-sided luminance gradients, as in Craik's (1940) version of the COCE. There are four stimulus configurations in Plate 7, whose corresponding luminance distributions are presented in Figure 3. The luminance distributions refer to horizontal (or equivalently, vertical) symmetry axes of the stimuli. The figures exhibit the four ways a closed contour can be formed with single-sided gradients. The gradients have the same amplitude but differ in polarity, that is, they are either positive (left column in Plate 7 and Figure 3) or negative (right column), and they also differ in location, that is, they are either on the inside (top row) or on the outside (bottom row) of the enclosed figure. These four varieties of COCE have not been systematically compared in parametric experiments. However, Heggelund and Krekling (1976) studied stimuli with positive inner gradients, Hamada $(1982,1985)$ studied stimuli with positive and stimuli with negative inner gradients, and Békésy (1972) studied stimuli with positive outer gradients.

Consider first the figures with outer gradients. The homogeneous square bordered by the positive outer gradient (Plate 7c) looks darker, and the one surrounded by the negative outer gradient (Plate 7d) looks brighter than the homogeneous background of equal luminance. This effect can be described as an instance of the COCE. However, it is also closely related to stimulus conditions

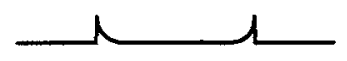

(a)

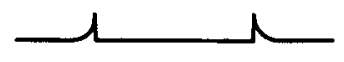

(c)

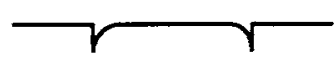

(b)

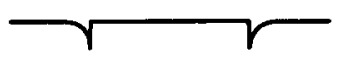

(d)
Figure 3. The huminance distributions of the four displays in Plate 7. (a) Positive inner gradients, (b) negative inner gradients, (c) positive outer gradients, and (d) negative outer gradients. 
and effects in brightness contrast. Both in Figure 3 and in brightness contrast displays, the stimulus is a small homogeneous region bordered by an abrupt luminance change; in both cases, the brightness of the percept is changed according to the polarity of the change. Similar stimuli inducing similar responses plausibly involve similar underlying neural processes.

Unlike figures with outer gradients, the central portions of the figures with inner gradients (Plates $7 \mathrm{a}$ and $7 \mathrm{~b}$ ) do not appear to be convincingly different in brightness from the surround. Although weak or absent in these particular displays, the COCE can be induced with stimulus distributions involving inner gradients, provided that luminance contrast is relatively small and retinal gradient width is relatively large (Hamada, 1985; Heggelund \& Krekling, 1976). However, with prolonged fixation, brightness phenomena analogous to the COCE can be induced in Plates $7 \mathrm{a}$ and $7 \mathrm{~b}$ as well. The enhancement of the COCE with fixation was noted by Jung (1978) and van den Brink and Keemink (1976). These effects will be discussed in the next section of this paper in the context of filling-in theories.

Note that in Cornsweet's (1970) version of the COCE, the border between the central disk and the surrounding annulus exhibits an inner and an outer gradient of opposite polarity, similar to Figure 1b. There are two versions of such a double-gradient stimulus configuration. One can be conceived of as a combination of Figures $3 \mathrm{a}$ and $3 \mathrm{~d}$, and the other as a combination of Figures $3 b$ and $3 c$. The asymmetry of the effects of the two types of gradients in single-gradient stimuli demonstrated in Plate 7 suggest that in double-gradient figures the two gradients may not contribute equally to the final brightness percept. This prediction could be empirically tested by parametric variation of the luminance contrast of each gradient in doublegradient figures.

\section{Filling-in Theories}

The guiding idea in this approach is that brightness and color of interiors of homogeneous regions are determined by a process of lateral spread of neural activation that is initiated by units responding to abrupt changes of luminance and wavelength. The best empirical evidence for the reality of such processes is provided by experiments with stabilized images. In such displays, the proximal stimulus distribution, or some portion of it, is projected at fixed retinal locations for extended periods of time. This is accomplished with the help of various technical devices. If the contour of a region is stabilized with respect to the retina in this manner, the region takes on the color and brightness of the surround. For example, if the borders of a red circle on a blue background are stabilized, the visual field becomes uniformly blue, despite the continuous presence of long-wave stimulation (Gerrits \& Vendrick, 1970; Krauskopf, 1963; Yarbus, 1957). The cause for these phenomena may be that continuous invariant stimulation causes desensitization and inactivation of units responding to edges. Their influence on neighbor- ing portions of the visual field would thus be eliminated, and the features of region interiors bounded by stabilized contours would instead be determined by conditions in the surround.

Another way to induce filling-in percepts by reducing the influence of edges is to blur them. In a demonstration by Krauskopf (1967), slides of a circle of one color on a surround of another were projected strongly out of focus. After a period of fixation, the central portion of such a display assumed the color of the surround, just as in stabilized image experiments. A black-and-white version of this effect was presented by Cornsweet (1970), and a related effect involving low-contrast shadows was described by Hering (1878/1964).

Gerrits and Vendrik (1970) presented a theory in which a neural filling-in process explains the perceptual fillingin phenomena. In this theory, a lower level of visual processing, composed of cells with antagonistic concentric receptive fields, is sensitive to contours. Information from this level is passed to a higher level, in which neural activities corresponding to region contours spread within region interiors. This theory was used to explain the appearance of stabilized images and scotoma, and did not explicitly discuss the COCE. However, it is clear that such processes may provide the neural foundation for the explanation of the COCE noted by Ratliff $(1971,1972)$, which postulates extrapolation of contour-induced response maxima and minima over neighboring regions.

Hamada $(1984,1985)$ has formulated a multistage model of brightness perception and applied it to the COCE. The model includes an edge-detecting stage involving zero-crossings, followed by a stage of averaging or filling-in activity within intervals located between zerocrossings. A theory involving neural filling-in processes was also developed by Grossberg and his associates (Cohen \& Grossberg, 1984; Grossberg, 1983, 1987a, 1987b; Grossberg \& Mingolla, 1985a, 1985b, 1987). The theory involves several stages of neural interactions of different types, and offers a unified approach to a large number of visual phenomena. Only the aspects of this theory that are relevant for the explanation of the brightness phenomena in the COCE will be briefly described here. Two contour-sensitive subsystems are postulated. One subsystem, called the Boundary Contour System, sensitive to edges but not to the direction of contrast, is involved in contour extraction and completion. Another subsystem, called the Feature Contour System, contains a level whose circularly concentric antagonistic units are also sensitive to contours but retain the information about contrast direction. Units at this level initiate activity at a subsequent level that contains units that support rapid lateral spread of neural activity. This spread is contained within regions delineated by boundaries created by the Boundary Contour System. These mechanisms explain the standard versions of the COCE. In addition, Cohen and Grossberg (1984) presented computer simulations of Hamada's (1982) and Coren's (1983) studies of COCE. One shortcoming of the theory is that filling-in was mathe- 
matically formulated as a one-dimensional process. However, this formulation has recently been extended to two dimensions, and the model has been successfully applied to simulate a number of brightness phenomena, including brightness contrast, brightness constancy, and some demonstrations presented in this paper (Grossberg \& Todorović, in press). For example, the absence of COCE in Plate 2 results, in the model, from the lack of boundaries which contain the spread of activity in Plate 1 . The difference in appearance between the occluded and unoccluded versions of a display similar to Plate 6 has also been simulated.

\section{Filling-in Demonstrations}

As noted, the displays that induce the perceptual fillingin effects involve stabilized or blurred contours. I will now discuss an additional, related way to induce fillingin, which is also relevant to the COCE, since it involves cusp-shaped brightness profiles. First, consider again the figures with inner gradients (Plates $7 \mathrm{a}$ and $7 \mathrm{~b}$ ). After a period, say $20 \mathrm{sec}$, of steady fixation under good illumination from close up, say $10 \mathrm{~cm}$, the luminance gradients in these figures become less noticeable, as the inner portions of the regions gradually change their brightness levels in accordance with their contours: the interior of the figure with positive inner gradients becomes brighter than the background, and the interior of the figure with negative inner gradients becomes darker. The phenomenon can be described as a filling-in of region interiors with brightness attributes of the border region. In addition, very steady fixation can lead to the brief disappearance of a figure, with the whole display assuming the homogeneous brightness level of the surround. All these effects are lost after eye movements or displacements of the display. Note that continuous fixation also induces the gradual disappearance of gradients and enhancement of the COCE in configurations with outer gradients (Plates $7 \mathrm{c}$ and 7d). The change in appearance of these stimuli during prolonged fixation is probably due in part to adaptation processes. However, this may not be the complete explanation, since adaptation is usually regarded to be a local phenomenon. Thus, it is not clear how such processes could cause brightness changes in opposite directions in interior portions with equal luminance levels in Plates $7 \mathrm{a}$ and $7 \mathrm{~b}$.

Plate 8 presents a variation of this way of induction of the filling-in phenomenon, demonstrating also the role of topological structure, as well as the influence of motion on brightness. The figure consists of four disconnected negative wedge-shaped gradients. The luminance profile is similar to the one in Figure 3d, but the four parts of the figure are radially "pulled apart," the left edge to the left, the right one to the right, and likewise for the top and bottom edges. If this figure is viewed from a greater distance, say a meter or two, the percept approximately agrees with the luminance distribution. However, viewed from a close distance, only a couple of seconds of gazing at the center should lead to an increase of the brightness homogeneity of the interior of the figure partially bounded by the four gradients, and make it look somewhat darker than the exterior. Thus, although the figure is not topologically closed, luminance conditions at the gradient edges influence the brightness of the interior. However, this influence is found to diminish and disappear in displays (not presented), in which the four gradients are radially pulled further apart from each other over increasing distances.

With this display, repeated production and annihilation of perceptual filling-in can be induced by the following operations. Shake the picture, that is, displace it to and fro with short, rapid, vigorous movements of the hand, keeping it at the same distance from the eyes. During the movement, the central portion of the display will brighten up, approximately to the level of the exterior. As soon as the movement stops, the brightness of the central portion will return to its previous lower level. Some observers describe the visual events following the stopping of the movement of Plate 8 as "a wave of darkness" filling in the region swiftly from the four gradient edges toward the center. To obtain a clear and convincing effect, one should repeat the shaking and stopping sequence several times, while constantly fixating the display. Brightness changes in the reverse direction are obtained in displays (not presented) with the same topology as Plate 8 but containing positive gradients. Note that, in addition to the brightness changes, the locations and widths of the four gradients in the picture appear to change during the movement. Similar, but less pronounced, changes can also be noticed with horizontal displacements of Plates 1 and 4 .

The different ways to induce the perceptual filling-in phenomenon can be qualitatively compared with respect to the manner of the spatial and temporal variation of the stimulus distribution. The normal viewing stimulation is characterized by moderate spatial variations, due to gradients and edges, and moderate temporal variation, due to involuntary eye movements. The stabilized vision stimuli exhibit moderate spatial variation but zero temporal variation. Stimuli with blurred contours, viewed with voluntary fixation, contain low spatial and temporal variation. Finally, Plates 7 and 8 contain moderate spatial variation; during fixation they exhibit low temporal variation, and during display movement they exhibit high temporal variation. Continuous fixation of Plate 8 , without total image stabilization, reduces the normal temporal rate of change of stimulation, but does not annihilate it completely, thus providing a smaller set of luminance and contrast-sensitive cells with longer than usual stimulation. Such stimulus conditions appear to favor the filling-in brightness effect. On the other hand, continuous largescale displacements of the display stimulate a greater number of neurons but, at least for a part of them, at a faster than optimal rate. Under such conditions, the effect of the luminance edges is temporally and spatially blurred. This appears to attenuate the filling-in process. These facts suggest that luminance adaptation and spatiotemporal resolution sensitivity, as well as the differences between sus- 
tained and transient cells, may play a role in the filling-in process. Clearly, parametric studies are needed of the influence of spatiotemporal stimulus variations on filling-in.

Cavanagh and Anstis (1986) reported brightness changes due to motion of stimuli with saw-tooth luminance profiles, similar to a conjunction of several gradients of a single type from Plate 8. The direction of brightness change in their stimuli depends on gradient polarity and motion direction, and involves the whole stimulus, which has no uniform regions. In contrast, Plate 8 contains nonconjuncted gradients of different polarities and orientations, the brightness effect does not appear to depend on motion direction, and it involves the uniform, gradientfree portion of the stimulus. Thus, it is not clear whether the same mechanisms are involved in both effects.

In sum, the thrust of the arguments and displays presented in this part is against a too literal analogy of mathematical integration and visual processes responsible for the COCE, and lends further support for the notion of the spread of brightness attributes from loci corresponding to abrupt luminance changes. Among the integration theories of the COCE, those that include a neural-like model, involving a set of computational cells working in parallel, have all invoked a mechanism analogous to lateral spread of excitation (Blake, 1985; Frisby, 1979; Horn, $1974,1986)$. Thus, a type of filling-in appears to be the preferred noncognitive mechanism for the explanation of the COCE. The most successful theory of the detailed structure of this process will be the one that is most consistent with all varieties of the COCE and related brightness phenomena, as well as the known physiological structure of the visual system.

\section{REFERENCES}

AREND, L. E. (1973). Spatial differential and integral operations in human vision: Implications of stabilized retinal image fading. Psychological Review, 80, 374-395.

AREND, L. (1985). Spatial gradient illusions and inconsistent integrals. Supplement to Investigative Ophthalmology \& Visual Science, 26, 280.

Arend, L. E., Buehler, J. N., \& LoCkHEAd, G. R. (1971). Difference information in brightness perception. Perception \& Psychophysics, 9, 367-370.

AREND, L. E., \& GolDsTern, R. E. (1987). Lightness models, gradient illusions and curl. Perception \& Psychophysics, 42, 65-80.

Baumgartner, G. (1960). Indirekte Grössenbestimmung der receptiven Felder der Retina beim Menschen mittels der Hermannschen Gittertäuschung (Abstract). Pflügers Archiv für die Gesamte Physiologie des Menschen und der Tiere, 272, 21-22.

BÉKÉSY, G. VON (1972). Compensation method to measure the contrast produced by contours. Journal of the Optical Society of America, 62, 1247-1251.

BENARY, W. (1924). Beobachtungen zu einem Experiment über Helligkeitskontrast. Psychologische Forschung, 5, 131-142. [Translated as "The influence of form on brightness contrast." In W. D. Ellis (Ed.), A source book of Gestalt psychology. London: Routledge \& Kegan Paul, 1938.]

BLAKE, A. (1985). Boundary conditions for lightness computation in Mondrian world. Computer Vision, Graphics, \& Image Processing, 14, 314-327.

BRIDGEMAN, B. (1983). Isomorphism is where you find it. Behavioral \& Brain Sciences, 6, 658-659.
BrindLey, G. (1970). Physiology of the retina and visual pathways (2nd ed.). Baltimore: Williams \& Wilkins.

Campbell, F. W., Howell, E. R., \& Johnstone, J. R. (1978). A comparison of threshold and suprathreshold appearance of gratings with components in the low and high spatial frequency range. Journal of Physiology, 284, 193-201.

CAmpbell, F. W., \& Robson, J. G. (1968). Application of Fourier analysis to the visibility of gratings. Journal of Physiology, 197, 551-566.

Cavanagh, P., \& Anstis, S. M. (1986). Brightness shift in drifting ramp gratings isolates a transient mechanism. Vision Research, 26, 899-908.

Cohen, M. A., \& Grossberg, S. (1984). Neural dynamics of brightness perception: Features, boundaries, diffusion, and resonance. Perception \& Psychophysics, 36, 428-456.

COREN, S. (1983). When "filling in" fails. Behavioral \& Brain Sciences, 6, 661-662.

Cornsweet, T. N. (1970). Visual perception. New York: Academic Press.

CraIK, K. J. W. (1940). Visual adaptation. Unpublished doctoral thesis, Cambridge University.

Davidson, M., \& Whiteside, J. A. (1971). Human brightness perception near sharp contours. Journal of the Optical Society of America, 61, 530-536.

DeValois, R. L., \& Pease, P. L. (1971). Contours and contrast: Responses of monkey lateral geniculate nucleus cells to luminance and color figures. Science, 171, 694-696.

DeValois, R. L., Webster, M. A., \& DeValois, K. K. (1986). Temporal properties of brightness and color induction. Vision Research, 26, 887-897.

Dooley, R. P., \& Greenfield, M. I. (1977). Measurements of edgeinduced visual contrast and a spatial-frequency interaction of the Cornsweet illusion. Journal of the Optical Society of America, 67, 761-765.

EHRENSTEIN, W. (1974). Über Abwandlungen der L. Hermannschen Helligkeitserscheinung. Zeitschrift für Psychologie, 150, 83-91.

Enroth-Cugell, C., \& Robson, J. G. (1966). The contrast sensitivity of retinal ganglion cells of the cat. Journal of Physiology, 187, 517-552.

Enroth-Cugell, C., \& Robson, J. G. (1984). Functional characteristics and diversity of cat retinal ganglion cells. Investigative Ophthalmology \& Visual Science, 25, 250-267.

EsCHER, M. C. (1961). The graphic work of M. C. Escher. London: Oldburne.

FoleY, J. M., \& MCCOURT, M. E. (1985). Visual grating induction. Journal of the Optical Society of America, A2, 1220-1230.

FosTER, D. H. (1983). Experimental test of a network theory of vision. Behavioral \& Brain Sciences, 6, 664-665.

FrisBY, J. P. (1979). Seeing: Illusion, brain and mind. Oxford: Oxford University Press.

FrY, G. A. (1948). Mechanisms subserving simultaneous brightness contrast. American Journal of Optometry \& Archives of the American Academy of Optometry, 25, 162-178.

FULD, K., \& O'DONNELL, K. (1984). Brightness matching and scaling of the Ehrenstein illusion. In L. Spillmann \& B. R. Wooten (Eds.), Sensory experience, adaptation and perception. Hillsdale, $\mathrm{NJ}$ : Erlbaum.

Gerrits, H. J. M., \& Vendrick, A. J. H. (1970). Simultaneous contrast, filling-in process, and information processing in man's visual system. Experimental Brain Research, 11, 411-430.

Gilchrist, A. (1977). Perceived lightness depends on perceived spatial arrangement. Science, 195, 185-187.

GrossBerg, S. (1983). The quantized geometry of visual space: The coherent computation of depth, form, and lightness. Behavioral \& Brain Sciences, 6, 625-692.

GrossBerg, S. (1987a). Cortical dynamics of three-dimensional form, color, and brightness perception, I: Monocular theory. Perception \& Psychophysics, 41, 87-116.

GrossBerG, S. (1987b). Cortical dynamics of three-dimensional form, color, and brightness perception, II: Binocular theory. Perception \& Psychophysics, 41, 117-158. 
Grossberg, S., \& Mingolla, E. (1985a). Neural dynamics of perceptual grouping: Textures, boundaries, and emergent segmentations. Perception \& Psychophysics, 38, 141-171.

Grossberg, S., \& Mingolla, E. (1985b). Neural dynamics of form perception: Boundary completion, illusory figures and neon color spreading. Psychological Review, 92, 173-211.

Grossberg, S., \& Mingolla, E. (1987). Neural dynamics of surface perception: Boundary webs, illuminants, and shape-from-shading. Computer Vision, Graphics, \& Image Processing, 37, 116-165.

GrossberG, S., \& ToDorović, D. (in press). Neural dynamics of 1-D and 2-D brightness perception: A unified model of classical and recent phenomena. Perception \& Psychophysics.

GrownEY, R. L., \& NerI, D. F. (1986). The appearance of the Cornsweet illusion: Measures of perceived contrast and evenness of brightness. Perception \& Psychophysics, 39, 81-86.

HAMADA, J. (1982). The contour enhancement effects produced by darkening effects. In H.-G. Geissler \& P. Petzold (Eds.), Psychophysical judgment and the process of perception. Amsterdam: North-Holland.

HAMADA, J. (1984). A multi-stage model for border contrast. Biological Cybernetics, 51, 65-70.

HAMADA, J. (1985). Asymmetric lightness cancellation in Craik-O'Brien patterns of negative and positive contrast. Biological Cybernetics, 52 , $117-122$.

HegGelund, P., \& Krekuing, S. (1976). Edge dependent lightness distributions at different adaptation levels. Vision Research, 16, 493-496.

HeinemanN, E. G. (1972). Simultaneous brightness induction. In D. Jameson \& L. M. Hurvich (Eds.), Handbook of sensory physiology: Vol. VII/4. Visual psychophysics. Berlin: Springer-Verlag.

Helson, H. (1963). Studies of anomalous contrast and assimilation. Journal of the Optical Society of America, 53, 179-184.

Hering, E. (1964). Outlines of a theory of light sense. Cambridge: Howard. (Original work published 1878)

HoRN, B. K. P. (1974). Determining lightness from an image. Computer Graphics \& Image Processing, 3, 277-299.

HORN, B. K. P. (1986). Robot vision. Cambridge, MA: MIT Press. HURLBERT, A. (1986). Formal connections between lightness algorithms. Journal of the Optical Society of America, A3, 1684-1693.

HuRvich, L. M. (1981). Color vision. Sunderland, MA: Sinauer.

IsoNo, H. (1979a). Measurement of edge-induced visual contrast (Note No. 233). NHK Laboratories.

Isono, H. (1979b). A reexamination of contrast threshold difference beween spatial sine-wave and square-wave gratings. Vision Research, 19, 603-607.

JuNG, R. (1978). Einführung in die Sehphysiologie. In O. H. Gauer, K. Kramer, \& R. Jung (Eds.), Physiologie des Menschen, Band 13. Sehen: Sinnesphysiologie III. München: Urban and Schwarzenberg.

Kanizsa, G. (1979). Organization in vision. New York: Praeger.

KatZ, D. (1935). The world of colour. London: Kegan Paul, Trench, Trubner.

KoFFKA, K. (1935). Principles of Gestalt psychology. New York: Harcourt, Brace.

KöHLER, W. (1929). Gestalt psychology. New York: Liveright.

KRAUSKOPF, J. (1963). Effect of retinal image stabilization on the appearance of heterochromatic targets. Journal of the Optical Society of America, 53, 741-744.

KRAUSKOPF, J. (1967). Heterochromatic stabilized images: A classroom demonstration. American Journal of Psychology, 80, 634-636.

LAMING, D. (1983). On the need for discipline in the construction of psychological theories. Behavioral \& Brain Sciences, 6, 669-670.

LAND, E. H. (1974). Smitty Stevens' test of retinex theory. In H. R. Moskowitz, B. Scharf, \& J. C. Stevens (Eds.), Sensation and measurement. Dordrecht: Reidel.

LAND, E. H. (1977). The retinex theory of color vision. Scientific American, 237, 108-128.

LAND, E. (1986). Recent advances in retinex theory. Vision Research, 26, 7-21.

LAND, E. H., \& MCCANN, J. J. (1971). Lightness and retinex theory. Joumal of the Optical Society of America, 61, 1-11.
MACH, E. (1865). Über die physiologische Wirkung der räumlichen Vertheilung des Lichtreizes auf die Netzhaut, I. Sitzungsbericht der Mathematisch-naturwissenschaftlichen Classe der Kaiserlichen Akademie der Wissenschaften, 52, 303-322.

Maffei, L., Morrone, C., Pirchio, M., \& Sandini, G. (1979). A perceptual phenomenon and its neurophysiological correlate. Perception, 88, 43-46.

MarR, D., \& Hildreth, E. C. (1980). Theory of edge detection. Proceedings of the Royal Society of London, Series B, 207, 187-217.

McCourT, M. E. (1982). A spatial frequency dependent gratinginduction effect. Vision Research, 22, 119-134.

MüLleR, G. E. (1896). Zur Psychophysik der Gesichtsempfindungen. Zeitschrift für Psychologie, 10, 1-82.

O'BRIEN, V. (1958). Contour perception, illusion, and reality. Journal of the Optical Society of America, 48, 112-119.

Penrose, L. S., \& Penrose, R. (1958). Impossible objects: A special type of visual illusion. British Journal of Psychology, 49, 31-33.

QuiNN, P. C. (1985). Suprathreshold contrast perception as a function of spatial frequency. Perception \& Psychophysics, 38, 408-414.

RATLIFF, F. (1965). Mach bands: Quantitative studies on neural networks in the retina. San Francisco: Holden-Day.

RATLIFF, F. (1971). Contour and contrast. Proceedings of the American Philosophical Society, 115, 150-163.

Ratliff, F. (1972). Contour and contrast. Scientific American, 226, 90-101.

RatlifF, F. (1978). A discourse on edges. In J. C. Armington, J. Krauskopf, \& B. R. Wooten (Eds.), Visual psychophysics and physiology. New York: Academic Press.

RatuifF, F., \& SiRovich, L. (1978). Equivalence classes of visual stimuli. Vision Research, 18, 845-851.

Redies, C., SpillmanN, L., \& Kunz, K. (1984). Colored neon flanks and line gap enhancement. Vision Research, 10, 1303-1309.

RoDIECK, R. W. (1965). Quantitative analysis of cat retinal ganglion cell response to visual stimuli. Vision Research, 5, 583-601.

SAKATA, H. (1981). Mechanism of Craik-O'Brien effect. Vision Research, 21, 693-699.

Schiffman, H., \& Crovitz, H. F. (1972). A two-stage model of brightness. Vision Research, 12, 2121-2131.

SHAPLEY, R., \& GoRDON, J. (1985). Nonlinearity in the peception of form. Perception \& Psychophysics, 37, 84-88.

ShaPley, R. M., \& TolhuRst, D. J. (1973). Edge detectors in human vision. Journal of Physiology, 229, 165-183.

SHEPARD, R. N. (1964). Circularity in judgment of relative pitch. Journal of the Acoustical Society of America, 36, 2346-2353.

Spillmann, L., \& LeVine, J. (1971). Contrast enhancement in a Hermann grid with variable figure-ground ratio. Experimental Brain Research, 13, 547-559.

Stevens, S. S. (1957). On the psychophysical law. Psychological Review, 64, 153-180.

Suluvan, G. D., \& Georgeson, M. A. (1977). The missing fundamental illusion: Variation of spatiotemporal characteristics with dark adaptation. Vision Research, 17, 977-981.

Teller, D. Y. (1980). Locus questions in visual science. In C. S. Harris (Ed.), Visual coding and adaptability. Hillsdale, NJ: Erlbaum.

Teller, D. Y. (1984). Linking propositions. Vision Research, 24, 1233-1246.

Teller, D. Y., \& PUGH, E. N., JR. (1983). Linking propositions in color vision. In J. D. Mollon \& L. T. Sharpe (Eds.), Colour vision. London: Academic Press.

Terzopoulos, D. (1986). Image analysis using multigrid relaxation methods. IEEE Transactions on Pattern Analysis \& Machine Intelligence, 8, 129-139.

TODORović, D. (1983). Brightness perception and the Craik-O'BrienCornsweet effect. Unpublished master's thesis, University of Connecticut. 
Tolnurst, D. J. (1972). On the possible existence of edge detector neurons in the human visual system. Vision Research, 12, 797-804.

UllmaN, S. (1984). Introduction. In S. Ullman \& W. Richards (Eds.), Image understanding 1984. Norwood, NJ: Ablex.

VAN DEN BRINK, G., \& KeEminK, C. J. (1976). Luminance gradients and edge effects. Vision Research, 16, 155-159.

VAN ESSEN, D. C. (1985). Functional organization of primate visual cortex. In A. Peters \& E. G. Jones (Eds.), Cerebral cortex (Vol. 3). New York: Academic Press.

WALLACH, H. (1948). Brightness constancy and the nature of achromatic colors. Journal of Experimental Psychology, 38, 310-324.

WALLS, G. (1954). The filling-in process. American Joumal of Optometry, 31, 329-340.
Ware, C., \& Cowan, W. B. (1983). The chromatic Cornsweet effect. Vision Research, 23, 1075-1077.

White, M. (1979). A new effect of pattern on perceived lightness. Perception, 8, 413-416.

YARBUS, A. L. (1957). The perception of an image fixed with respect to the retina. Biophysics, 2, 683-690.

Yund, E. W., \& ARMINGTON, J. C. (1975). Color and brightness contrast effects as a function of spatial variables. Vision Research, 15 , 917-930.

(Manuscript received December 10, 1986; revision accepted for publication May 22, 1987.) 